\title{
Photon-Photon Physics in Very Peripheral Collisions of Relativistic Heavy Ions
}

\author{
Gerhard Baur*, \\ Institut für Kernphysik, Forschungszentrum Jülich, \\ Postfach 1913, D-52425 Jülich \\ Kai Hencken ${ }^{\dagger}$ and Dirk Trautmann; \\ Institut für Physik, Universität Basel, \\ Klingelbergstr. 82, CH-4056 Basel \\ February 1, 2008 \\ Topical Review, to appear in Journal of Physics G
}

\begin{abstract}
In central collisions at relativistic heavy ion colliders like the Relativistic Heavy Ion Collider RHIC/Brookhaven and the Large Hadron Collider LHC (in its heavy ion mode) at CERN/Geneva, one aims at detecting a new form of hadronic matter - the Quark Gluon Plasma. It is the purpose of this review to discuss a complementary aspect of these collisions, the very peripheral ones. Due to coherence, there are strong electromagnetic fields of short duration in such collisions. They give rise to photon-photon and photon-nucleus collisions with high flux up to an invariant mass region hitherto unexplored experimentally. After a general survey photon-photon luminosities in relativistic heavy ion collisions are discussed. Special care is taken to include the effects of strong interactions and nuclear size. Then photon-photon physics at various $\gamma \gamma$-invariant mass scales is discussed. The region of several $\mathrm{GeV}$, relevant for RHIC is dominated by QCD phenomena (meson and vector meson pair production). Invariant masses of up to about $100 \mathrm{GeV}$ can be reached at LHC, and the potential for new physics is discussed. Photonuclear reactions and other important background effects, especially diffractive processes are also discussed. A special chapter is devoted to lepton-pair production, especially electron-positron pair production; due to the strong fields new phenomena, especially multiple $e^{+} e^{-}$pair production, will occur there.
\end{abstract}

\section{Contents}

\begin{tabular}{lll}
\hline Introduction & 2
\end{tabular}

2 General survey of peripheral collisions 5

3 From impact-parameter dependent equivalent photon spectra to $\gamma \gamma$-luminosities $\quad 8$

3.1 Elastic vertices . . . . . . . . . . . . . . . . . 8

3.2 Inelastic Vertices and Higher Order Corrections . . . . . . . . . . . . 13

*EMail:G.Baur@fz-juelich.de

${ }^{\dagger}$ EMail:hencken@quasar.physik.unibas.ch

‡EMail:trautmann@ubaclu.unibas.ch 


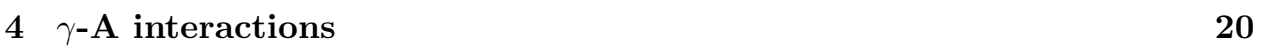

5 Photon-Photon Physics at various invariant mass scales 24

5.1 Basic QCD phenomena in $\gamma \gamma$-collisions . . . . . . . . . . . . 24

5.1.1 Hadron spectroscopy: Light quark spectroscopy . . . . . . . . . 24

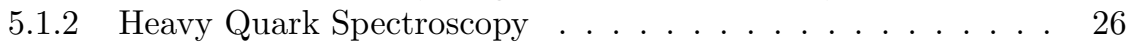

$5.1 .3 \quad$ Vector-meson pair production. Total hadronic cross-section . 26

$5.2 \gamma \gamma$-collisions as a tool for new physics . . . . . . . . . . . 27

$6 \quad$ Diffractive processes as background 29

7 Electron-Positron Pair production and QED of strong fields $\quad 29$

7.1 Free pair production, Strong field effects and multiple pair production 30

7.2 Bound-Free Pair Production . . . . . . . . . . . . . 33

8 Conclusion $\quad 34$

9 Acknowledgments

\section{Introduction}

The parton picture is very useful to study scattering processes at very high energies. In this model the scattering is described as an incoherent superposition of the scattering of the various constituents. For example, nuclei consist of nucleons which in turn consist of quarks and gluons, photons consist of lepton pairs, electrons consist of photons, etc. It is the subject of this topical review to discuss that relativistic nuclei have photons as an important constituent, especially for low enough virtuality $Q^{2}=-q^{2}>0$ of the photon. This is due to the coherent action of all the charges in the nucleus. The virtuality of the photon is related to the size $R$ of the nucleus by

$$
Q^{2} \lesssim 1 / R^{2}
$$

the condition for coherence. The radius of a nucleus is given approximately by $R=1.2 \mathrm{fm} A^{1 / 3}$, where $A$ is the nucleon number. From the kinematics of the process one has

$$
Q^{2}=\frac{\omega^{2}}{\gamma^{2}}+Q_{\perp}^{2}
$$

Due to the coherence condition the maximum energy of the quasireal photon is therefore given by

$$
\omega_{\max } \approx \frac{\gamma}{R}
$$

and the maximum value of the perpendicular component is given by

$$
Q_{\perp} \lesssim \frac{1}{R}
$$

We define the ratio $x=\omega / E$, where $E$ denotes the energy of the nucleus $E=M_{N} \gamma A$ and $M_{N}$ is the nucleon mass. It is therefore smaller than

$$
x_{\max }=\frac{1}{R M_{N} A}=\frac{\lambda_{C}(A)}{R},
$$

where $\lambda_{C}(A)$ is the Compton wave length of the ion. Here and also throughout the rest of the paper we use natural units, setting $\hbar=c=1$.

The collisions of $e^{+}$and $e^{-}$has been the traditional way to study $\gamma \gamma$-collisions. Similarly photon-photon collisions can also be observed in hadron-hadron collisions. 
Since the photon number scales with $Z^{2}$ ( $Z$ being the charge number of the nucleus) such effects can be particularly large. Of course, the strong interaction of the two nuclei has to be taken into consideration.

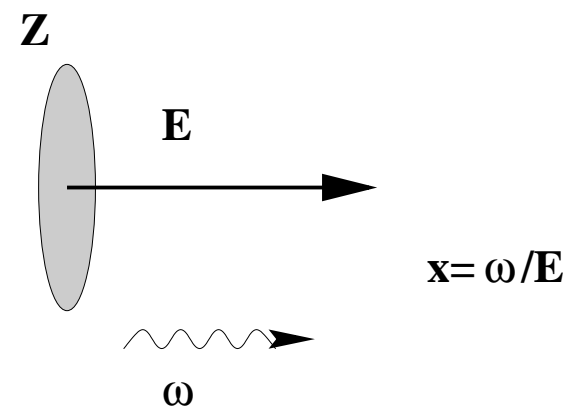

Figure 1: A fast moving nucleus with charge Ze is surrounded by a strong electromagnetic field. This can be viewed as a cloud of virtual photons. These photons can often be considered as real. They are called equivalent or quasireal photons. The ratio of the photon energy $\omega$ and the incident beam energy $E$ is denoted by $x=\omega / E$. Its maximal value is restricted by the coherence condition to $x<\lambda_{C}(A) / R \approx 0.175 / A^{4 / 3}$, that is, $x \lesssim 10^{-3}$ for $C a$ ions and $x \lesssim 10^{-4}$ for $\mathrm{Pb}$ ions.

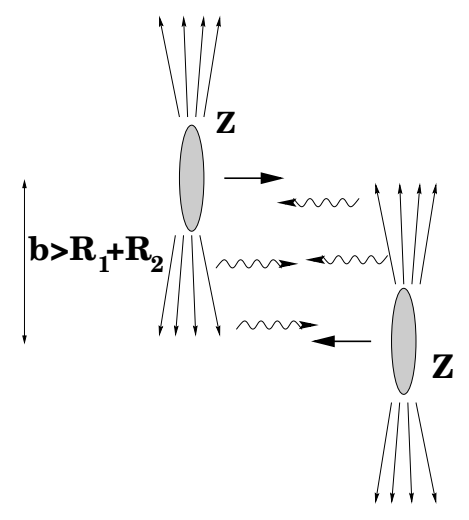

Figure 2: Two fast moving electrically charged objects are an abundant source of (quasireal) photons. They can collide with each other and with the other nucleus. For peripheral collisions with impact parameters $b>2 R$, this is useful for photonphoton as well as photon-nucleus collisions.

The equivalent photon flux present in medium and high energy nuclear collisions is very high, and has found many useful applications in nuclear physics [1], nuclear

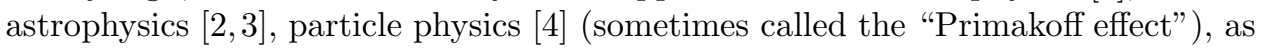
well as, atomic physics [. [0. It is the main purpose of this review to discuss the physics of photon-photon and photon-hadron (nucleus) collisions in high energy heavy ion collisions. With the construction of the "Relativistic Heavy Ion Collider" (RHIC) and the "Large Hadron Collider" (LHC) scheduled for 1999 and for 2004/2008, respectively, one will be able to investigate such collisions experimentally. The main purpose of these heavy ion colliders is the formation and detection of the quark-gluon-plasma, a new form of highly excited dense hadronic matter. Such 
a state of matter will be created in central collisions. The present interest is in the "very peripheral (distant) collisions", where the nuclei do not interact strongly with each other. From this point of view, grazing collisions and central collisions are considered as a background. It is needless to say that this "background" can also be interesting physics of its own.

The equivalent photon spectrum extends up to several $\mathrm{GeV}$ at RHIC energies $(\gamma \approx 100)$ and up to about $100 \mathrm{GeV}$ at LHC energies $(\gamma \approx 3000)$, see Eq. (3). Therefore the range of invariant masses $M_{\gamma \gamma}$ at RHIC will be up to about the mass of the $\eta_{c}$, at LHC it will extend into an invariant mass range hitherto unexplored.

We discuss the equivalent photon spectra of strongly interacting particles, from which $\gamma \gamma$-luminosities are obtained. Due to the coherence effect, the corresponding $\gamma \gamma$-luminosity is very high. In addition higher order and inelastic processes, which may occur in heavy ion collisions are discussed. Then the various possibilities for $\gamma \gamma$-physics in the different invariant mass regions will be explored. A special case of $\gamma \gamma$-physics is lepton pair production, in particular the $e^{+} e^{-}$pair production. Since the equivalent photon approximation fails in some regions of phase space, this case is discussed separately. Due to its strong-field aspects, the production of multiple $e^{+} e^{-}$-pairs is of basic interest. Electron-positron pair creation is also of practical interest due to the possibility of capturing an electron in a K,L,.. shell. This changes the charge state of the ion and leads to a beam loss and thus to a decrease in the luminosity. The very large cross section for the production of $e^{+} e^{-}$pairs (sometimes called QED-electrons) is also an important background for detectors.

Relativistic heavy ion collisions have been suggested as a general tool for two photon physics about a decade ago. Yet the study of a special case, the production of $e^{+} e^{-}$pairs in nucleus-nucleus collisions, goes back to the work of Landau and Lifschitz in 1934 [6] (In those days, of course, one thought more about high energy cosmic ray nuclei than relativistic heavy ion colliders). In the meantime the importance of this process has become very clear, and many studies followed, e.g., [7 9]. This subject will be dealt with in detail in Sec. 5 and 7, where also recent experimental results will be mentioned.

The general possibilities and characteristic features of two-photon physics in relativistic heavy ion collisions have been discussed in $[9$. The possibility to produce a Higgs boson via $\gamma \gamma$-fusion was suggested in 10,11]. In these papers the effect of strong absorption in heavy ion collisions was not taken into account. This absorption is a feature, which is quite different from the two-photon physics at $e^{+} e^{-}$ colliders. The problem of taking strong interactions into account was solved by using impact parameter space methods in 12 14. Thus the calculation of $\gamma \gamma$-luminosities in heavy ion collisions is put on a firm basis and rather definite conclusions were reached by many groups working in the field [15]; for a recent review containing further references see [16]. Subsequent studies - to be described in detail in this review - revealed in a clear way that the theoretical situation is basically understood. This opens the way for many interesting applications. Up to now hadronhadron collisions have not been used for two-photon physics. An exception can be found in [17]. There the production of $\mu^{+} \mu^{-}$pairs at the ISR was observed. The special class of events was selected, where no hadrons are seen associated with the muon pair in a large solid angle vertex detector. In this way one makes sure that the hadrons do not interact strongly with each other, i.e., one is dealing with peripheral collisions (with impact parameters $b>2 R$ ); the photon-photon collisions manifest themselves as "silent events".

We feel that this is a very good basis for planning concrete experiments, as it is done at RHIC 18 21] and LHC [22 24]. This review aims at giving the main physical ideas and provide the key formula and results. Details can be found in the literature. A few new results will also be presented. But the main emphasis is to discuss the principle ideas and results in the field. We hope that this review will 
further stimulate future investigations. It is appropriate to recall that RHIC will start operating in 1999, only a year from now.

\section{General survey of peripheral collisions}

Let us first discuss the importance of electromagnetic interactions in peripheral collisions for the case of elastic scattering. The strength of the Coulomb interaction is measured by the Coulomb (or Sommerfeld) parameter $\eta$ which is given in terms of the nuclear charges $Z_{1}$ and $Z_{2}$ by

$$
\eta=\frac{Z_{1} Z_{2} e^{2}}{\hbar v}
$$

For (ultra)relativistic collisions we have $v \approx c$ and thus $\eta \approx Z_{1} Z_{2} / 137$. Therefore for $p p$-collisions we have always $\eta \ll 1$ and the Born approximation, that is, one-photon exchange, is applicable. For this case, i.e., for $\eta \approx 1 / 137$, elastic scattering is reviewed, e.g., in [25]. (Experimental result for $p \bar{p}$ scattering at $\sqrt{s}=546$ and $1800 \mathrm{GeV}$ at Fermilab are given in 26 28]). There is a superposition of nuclear and Coulomb amplitudes and the elastic differential scattering cross section is divided into three distinct regions, separated by the value of the square of the momentum transfer $t$. It is defined as usual as $t=\left(p_{i}-p_{f}\right)^{2}$, which is negative (space-like momentum transfer) in our metric used. For $|t| \ll|t|_{\text {int }}$ Coulomb scattering dominates, for $|t| \gg|t|_{\text {int }}$, nuclear scattering dominates and for $|t| \approx|t|_{\text {int }}$ there is Coulomb-nuclear interference. $|t|_{\text {int }}$ is given by (see Eq. (3.13) of [25], where the factor of $Z_{1} Z_{2}$ is added for the scattering of two nuclei with charge $Z_{1}, Z_{2}$ instead of two protons):

$$
|t|_{\text {int }} \approx \frac{8 \pi \alpha Z_{1} Z_{2}}{\sigma_{\text {tot }}}
$$

The nuclear elastic scattering amplitude is usually parameterized as

$$
F_{n}=\frac{(\rho+i) \sigma_{t o t}}{4 \sqrt{\pi}} e^{B t / 2}
$$

where $\sigma_{\text {tot }}$ is the total cross section, $\rho=\frac{\operatorname{Re} f_{c . m .}(0)}{\operatorname{Im} f_{c . m .}(0)}$ and $B$ is a slope parameter related to the size of the hadron. In this normalization the differential cross section is given by

$$
\frac{d \sigma}{d t}=\left|F_{n}\right|^{2}
$$

On the other hand for (very) heavy ions we have $\eta \gg 1$ and semi-classical methods are appropriate to deal with elastic scattering (see also [29]). For $\eta \gg 1$ the Coulomb interaction is very strong and the Born approximation is no longer valid. (In an analogy with optics there is now Fraunhofer diffraction instead of Fresnel diffraction). Instead one should use a Glauber approximation, where the Coulomb interaction is taken into account to all orders. One can say that many photons are typically exchanged in the elastic collisions (in contrast to the Born approximation relevant for the $p p$ case, where one photon exchange is sufficient). One can now integrate over the impact parameter $b$ using the saddle point approximation. Thus one recovers the classical picture of scattering. The particles move essentially on a straight line with a constant velocity and an impact parameter $b$. For $Z_{1}=Z_{2}=Z$ at grazing impact parameter $b=2 R$ the momentum transfer is given by (see, e.g., [30])

$$
|t|_{\text {Coul }}=(\Delta p)^{2}=\left(\frac{2 Z^{2} e^{2}}{2 R v}\right)^{2} \approx \frac{Z^{4} e^{4}}{R^{2} c^{2}}
$$


This momentum transfer determines the scattering angle $\theta=\Delta p / p$, where $p$ is the momentum of the particle.

Let us compare this quantity with the corresponding one for $p p$-scattering, Eq. (7). With $\sigma_{\text {tot }} \approx 8 \pi R^{2}$ one has

$$
|t|_{\text {int }}=\frac{\alpha Z^{2}}{R^{2}}
$$

to be compared to

$$
|t|_{\text {Coul }}=\frac{\alpha Z^{2}}{R^{2}}\left(\alpha Z^{2}\right) .
$$

It seems interesting to note that $|t|_{\text {Coul }}$ is $Z^{2} \alpha$ times the corresponding quantity $|t|_{\text {int }}$. Evidently the corresponding scattering angles are exceedingly small. For $\eta \approx 1$,i.e., for $Z \approx 12$, the quantities $|t|_{\text {int }}$ and $|t|_{\text {Coul }}$ (Eq. (11) and (12)) are about equal, as should be the case. For such values of $Z$ the change from Fresnel to Fraunhofer scattering takes place. A more detailed discussion is given in Ch. 5.3.4 of [31], based on the work of [32].

The $p p$ cross section is rising with energy (see, e.g., p.193 of [33]). At LHC energies it will be of the order of $80 \mathrm{mb}$. What does this mean for $p$ - $\mathrm{A}$ and $\mathrm{A}$ A cross section? A discussion is given in [34]. Experimental information can be obtained from cosmic ray data for $p$-A reactions. Calculation for cross section for A-A data often make use of a density-folding approach. This approach can be justified starting from Glauber theory [35, 36]. One uses the thickness function $T_{A}(b)$ of each nucleus, which is defined as the projection of the density along the beam axis:

$$
T_{A}(b)=\int_{-\infty}^{+\infty} d z n_{A}\left(\sqrt{b^{2}+z^{2}}\right) .
$$

Here the nuclear density $n_{A}(r)$ is normalized to $\int d^{3} r n_{A}(r)=A$. From this one gets the "overlap function" given by

$$
T_{A B}(b)=\int d^{2} b_{1} \int d^{2} b_{2} t\left(\vec{b}-\vec{b}_{1}-\vec{b}_{2}\right) T_{A}\left(b_{1}\right) T_{B}\left(b_{2}\right),
$$

where $\vec{b}$ is the impact parameter between the two ions (see also Fig. 3).

$t(\vec{s})$ describes the finite range of the nucleon-nucleon interaction. It is proportional to the profile function $\Gamma(b)$ in the Glauber theory, but it is normalized to $\int d^{2} s t(\vec{s})=1$. Therefore we have $\int d^{2} b T_{A B}(b)=A B$. For high energies the NN cross section rises beyond the geometrical size of the protons. The finite range of the nucleon-nucleon interaction is important then. A similar situation occurs also in nuclear physics at lower energies, see, e.g., Figs. 2 and 3 of [37], where the influence of the finite range of the $\mathrm{NN}$ interaction is clearly seen on the total cross-section for scattering of $\mathrm{Li}$ and Be isotopes.

If the nucleon-nucleon cross section is almost purely absorptive, the scattering amplitude is almost imaginary and one can get $t(b)$ from the elastic differential cross section

$$
t(\vec{s})=\frac{\int d^{2} p_{\perp} \exp \left(i \vec{p}_{\perp} \vec{s}\right) \sqrt{\frac{d \sigma}{d^{2} p_{\perp}}}}{\left.(2 \pi)^{2} \sqrt{\frac{d \sigma}{d^{2} p_{\perp}}}\right|_{p_{\perp}=0}} .
$$

with $\frac{d \sigma}{d^{2} p_{\perp}}$ the elastic nucleon-nucleon cross section. Often $t(\vec{b})$ is approximated by an exponential function of the form $t(\vec{b}) \sim \exp \left(-\left(b / b_{0}\right)^{2}\right)$, where $b_{0}$ is the range of the interaction. 
If one can neglect the finite range of the interaction compared to the geometrical size of the nuclei, the "thickness function" can be simplified:

$$
\begin{aligned}
T_{A B}(b) & =\int d^{2} b_{1} \int d^{2} b_{2} \delta^{(2)}\left(b-b_{1}-b_{2}\right) T_{A}\left(b_{1}\right) T_{B}\left(b_{2}\right) \\
& =\int d^{2} b_{1} T_{A}\left(b_{1}\right) T_{B}\left(b-b_{1}\right)
\end{aligned}
$$

The inelastic scattering cross section of the nucleus-nucleus collision is given by

$$
\sigma_{A B, \text { inel }}=\int d^{2} b\left[1-\exp \left(-\sigma_{N N} T_{A B}(b)\right)\right]
$$

where $\sigma_{N N}$ is the total nucleon-nucleon cross section. This integration over $b$ allows the integrand to be interpreted as a probability for the two ions to interact. This probability is about 1 for small impact parameters, where the nuclei overlap, it drops to zero for large impact parameters. The width of the area, where it falls off, is given by the surface diffuseness of the nuclear densities and the range of the nucleon-nucleon interaction as given by $t$. Similarly the elastic cross section is given by

$$
\sigma_{A B, e l}=\int d^{2} b\left[1-\exp \left(-\sigma_{N N} T_{A B}(b) / 2\right)\right]^{2} .
$$

Using results from Regge theory (see, e.g., [38, 39]) one can estimate the range $b_{0}$ of the interaction by

$$
b_{0}=2 \sqrt{B_{0, p P}+\ln \left(s / s_{0}\right) \alpha_{P}^{\prime}} .
$$

Using for $B_{0, p P} \approx 2.4 \mathrm{GeV}^{-2}$ and for $\alpha_{P}^{\prime} \approx 0.25 \mathrm{GeV}^{-2}$, as found in [38, 39], one gets for LHC energies $\left(s=(2 \times 7 \mathrm{TeV})^{2}, s_{0}=1 \mathrm{GeV}^{2}\right) b_{0} \approx 1 \mathrm{fm}$, which is small compared to the nuclear radius.

As we will see in the next section, the photon-photon luminosity depends also on the probability for the two nuclei to interact with each other. Only those processes, where the ions do not interact, are useful for photon-photon physics. From our discussion here, we can conclude that the effect of the increasing range of the NN interaction will only be of some importance at the very high end of the invariant $\gamma \gamma$-masses for a quantitative determination of the photon-photon luminosity.

a) side view

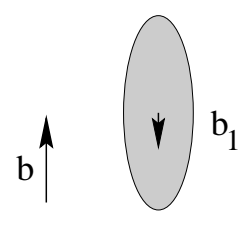

b) head on view

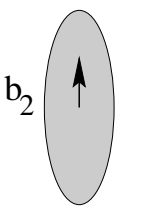

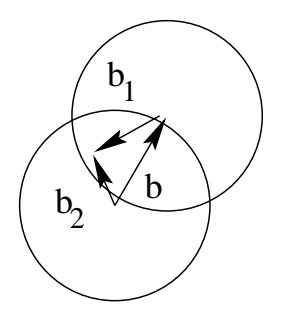

Figure 3: The probability of the nucleus to interact in central collisions can be described within the $\rho-\rho$ folding approach to the Glauber theory. The parameters b, $b_{1}$ and $b_{2}$ used in the text are explained. 


\section{From impact-parameter dependent equivalent photon spectra to $\gamma \gamma$-luminosities}

Photon-photon collisions have been studied extensively at $e^{+} e^{-}$colliders. The theoretical framework is reviewed, e.g., in [40]. The basic graph for the two-photon process in ion-ion collisions is shown in Fig. 1 . Two virtual (space-like) photons collide to form a final state $f$. In the equivalent photon approximation it is assumed that the square of the 4 -momentum of the virtual photons is small, i.e., $q_{1}^{2} \approx q_{2}^{2} \approx 0$ and the photons can be treated as quasireal. In this case the $\gamma \gamma$-production is factorized into an elementary cross section for the process $\gamma+\gamma \rightarrow f$ (with real photons, i.e., $q^{2}=0$ ) and a $\gamma \gamma$-luminosity function. In contrast to the pointlike elementary electrons (positrons), nuclei are extended, strongly interacting objects with internal structure. This gives rise to modifications in the theoretical treatment of two photon processes.

\subsection{Elastic vertices}

The emission of a photon depends on the (elastic) form factor. Often a Gaussian form factor or one of a homogeneous charged sphere is used. The typical behavior of a form factor is

$$
f\left(q^{2}\right) \approx\left\{\begin{array}{ll}
Z & \text { for }\left|q^{2}\right|<\frac{1}{R^{2}} \\
0 & \text { for }\left|q^{2}\right| \gg \frac{1}{R^{2}}
\end{array} .\right.
$$

For low $\left|q^{2}\right|$ all the protons inside the nucleus act coherently, whereas for $\left|q^{2}\right| \gg 1 / R^{2}$ the form factor is very small, close to 0 . For a medium size nucleus with, say, $R=5$ $\mathrm{fm}$, the limiting $Q^{2}=-q^{2}=1 / R^{2}$ is given by $Q^{2}=(40 \mathrm{MeV})^{2}=1.6 \times 10^{-3} \mathrm{GeV}^{2}$. Apart from $e^{+} e^{-}$(and to a certain extent also $\mu^{+} \mu^{-}$) pair production - which will be discussed separately below - this scale is much smaller than typical scales in the two-photon processes. Therefore the virtual photons in relativistic heavy ion collisions can be treated as quasireal. This is a limitation as compared to $e^{+} e^{-}$ collisions, where the two-photon processes can also be studied as a function of the corresponding masses $q_{1}^{2}$ and $q_{2}^{2}$ of the exchanged photon ("tagged mode").

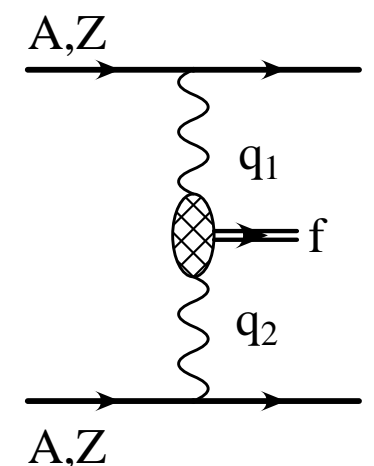

Figure 4: The general Feynman diagram of photon-photon processes in heavy ion collisions: Two (virtual) photons fuse in a charged particle collision into a final system $f$.

As was discussed already in the previous section, relativistic heavy ions interact strongly when the impact parameter is smaller than the sum of the radii of the 


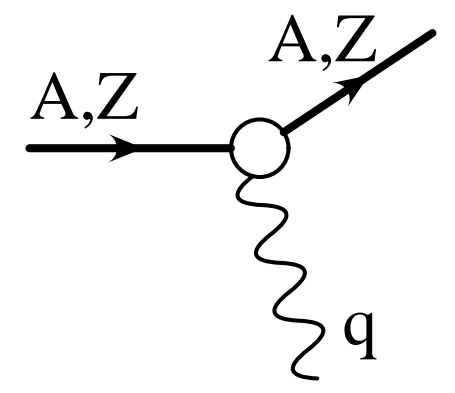

Figure 5: "Elastic photon emission": In order for the photon to interact coherently with the whole nucleus, its virtuality $Q^{2}$ is restricted to $Q^{2} \lesssim 1 / R^{2}$. In the calculation this is incorporated by the elastic form factor $f\left(q^{2}\right)$. This is important for a treatment, where plane waves are used. In the semiclassical (or Glauber) method, the detailed form of $f\left(q^{2}\right)$ is not relevant for collisions with $b>R$ (see text).

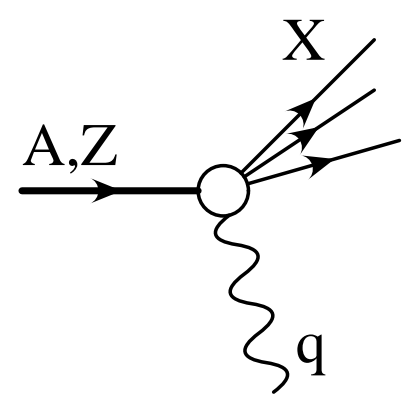

Figure 6: "Inelastic photon emission": As a nucleus is a rather weakly bound system, photon emission can lead to its breakup or excitation, especially for $Q^{2} \gtrsim 1 / R^{2}$. Also for this incoherent case an equivalent photon number, similar to the coherent case, can be defined. 
two nuclei. In such cases $\gamma \gamma$-processes are still present and are a background that has to be considered in central collisions. In order to study "clean" photon-photon events however, they have to be eliminated in the calculation of photon-photon luminosities as the particle production due to the strong interaction dominates. In the usual treatment of photon-photon processes in $e^{+} e^{-}$collisions plane waves are used and there is no direct information on the impact parameter. For heavy ion collisions on the other hand it is very appropriate to introduce impact parameter dependent equivalent photon numbers. They have been widely discussed in the literature (see e.g. [1, 30, 41])

The equivalent photon spectrum corresponding to a point charge $Z e$, moving with a velocity $v$ at impact parameter $b$ is given by

$$
N(\omega, b)=\frac{Z^{2} \alpha}{\pi^{2}} \frac{1}{b^{2}}\left(\frac{c}{v}\right)^{2} x^{2}\left[K_{1}^{2}(x)+\frac{1}{\gamma^{2}} K_{0}^{2}(x)\right],
$$

where $K_{n}(x)$ are the modified Bessel Functions (MacDonald Functions) [42] and $x=\frac{\omega b}{\gamma v}$. Then one obtains the probability for a certain electromagnetic process to occur in terms of the same process generated by an equivalent pulse of light as

$$
P(b)=\int \frac{d \omega}{\omega} N(\omega, b) \sigma_{\gamma}(\omega) .
$$

Possible modifications of $N(\omega, b)$ due to an extended spherically symmetric charge distribution are given in 433 (see also Eq. (49) below). It should be noted that Eq. (22) also describes the equivalent photon spectrum of an extended charge distribution, such as a nucleus, as long as $b$ is larger than the extension of the object. This is due to the fact that the electric field of a spherically symmetric system depends only on the total charge, which is inside it. As one often wants to avoid also final state interaction between the produced system and the nuclei, one has to restrict oneself to $b_{i}>R_{i}$ and therefore the form factor is not very important. For inelastic vertices a photon number $N(\omega, b)$ can also be defined, as will be discussed in Sec. 3.2 below.

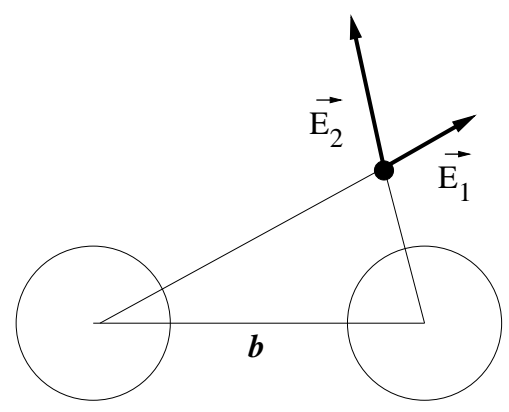

Figure 7: View of the collision perpendicular to the beam direction: The electric field vector points along the direction of the individual impact parameter.

As the term $x^{2}\left[K_{1}^{2}(x)+1 / \gamma^{2} K_{0}^{2}(x)\right]$ in Eq. (22) can be roughly approximated as 1 for $x<1$ and 0 for $x>1$, so that the equivalent photon number $N(\omega, b)$ is almost a constant up to a maximum $\omega_{\max }=\gamma / b(x=1)$. By integrating the photon spectrum (Eq. (22)) over $b$ from a minimum value of $R_{\text {min }}$ up to infinity (where essentially only impact parameter up to $b_{\max } \approx \gamma / \omega$ contribute, compare with Eq. (3)), one can define an equivalent photon number $n(\omega)$. This integral can 
be carried out analytically and is given by [1, 30]

$$
n(\omega)=\int d^{2} b N(\omega, b)=\frac{2}{\pi} Z_{1}^{2} \alpha\left(\frac{c}{v}\right)^{2}\left[\xi K_{0} K_{1}-\frac{v^{2} \xi^{2}}{2 c^{2}}\left(K_{1}^{2}-K_{0}^{2}\right)\right],
$$

where the argument of the modified Bessel functions is $\xi=\frac{\omega R_{\min }}{\gamma v}$. The cross section for a certain electromagnetic process is then

$$
\sigma=\int \frac{d \omega}{\omega} n(\omega) \sigma_{\gamma}(\omega)
$$

Using the approximation above for the MacDonald functions, we get an approximated form, which is quite reasonable and is useful for estimates:

$$
n(\omega) \approx \frac{2 Z^{2} \alpha}{\pi} \ln \frac{\gamma}{\omega R_{\min }} .
$$

The photon-photon production cross-section is obtained in a similar factorized form, by folding the corresponding equivalent photon spectra of the two colliding heavy ions 13, 14] (for polarization effects see [13], they are neglected here)

$$
\sigma_{c}=\int \frac{d \omega_{1}}{\omega_{1}} \int \frac{d \omega_{2}}{\omega_{2}} F\left(\omega_{1}, \omega_{2}\right) \sigma_{\gamma \gamma}\left(W_{\gamma \gamma}=\sqrt{4 \omega_{1} \omega_{2}}\right),
$$

with

$$
\begin{aligned}
F\left(\omega_{1}, \omega_{2}\right)= & 2 \pi \int_{R_{1}}^{\infty} b_{1} d b_{1} \int_{R_{2}}^{\infty} b_{2} d b_{2} \int_{0}^{2 \pi} d \phi \\
& \times N\left(\omega_{1}, b_{1}\right) N\left(\omega_{2}, b_{2}\right) \Theta\left(b_{1}^{2}+b_{2}^{2}-2 b_{1} b_{2} \cos \phi-R_{\text {cutof } f}^{2}\right),
\end{aligned}
$$

$\left(R_{\text {cutoff }}=R_{1}+R_{2}\right)$. This can also be rewritten in terms of the invariant mass $W_{\gamma \gamma}=\sqrt{4 \omega_{1} \omega_{2}}$ and the rapidity $Y=1 / 2 \ln \left(\left(P_{0}+P_{z}\right) /\left(P_{0}-P_{z}\right)\right)=1 / 2 \ln \left(\omega_{1} / \omega_{2}\right)$ as:

$$
\sigma_{c}=\int d W_{\gamma \gamma} d Y \frac{d^{2} L}{d W_{\gamma \gamma} d Y} \sigma_{\gamma \gamma}\left(W_{\gamma \gamma}\right)
$$

with

$$
\frac{d^{2} L_{\gamma \gamma}}{d W_{\gamma \gamma} d Y}=\frac{2}{W_{\gamma \gamma}} F\left(\frac{W_{\gamma \gamma}}{2} e^{Y}, \frac{W_{\gamma \gamma}}{2} e^{-Y}\right) .
$$

Here energy and momentum in the beam direction are denoted by $P_{0}$ and $P_{z}$. The transverse momentum is of the order of $P_{\perp} \leq 1 / R$ and is neglected here. The transverse momentum distribution is calculated in 44 .

In [44] and 45] this intuitively plausible formula is derived ab initio, starting from the assumption that the two ions move on a straight line with impact parameter b. Eqs. (27) and (29) are the basic formulae for $\gamma \gamma$-physics in relativistic heavyion collisions. The advantage of heavy nuclei is seen in the coherence factor $Z_{1}^{2} Z_{2}^{2}$ contained in Eqs. (27)-(30).

Let us make a few remarks: In Eq. (28) a sharp cut-off in impact parameter space at $b_{\text {min }}=R_{\text {cutoff }}$ is introduced. There is some ambiguity in the numerical value of $R_{\text {cutoff }}$ : As was discussed in Sec. 2 the total cross section for nucleonnucleon collisions is rising with energy. To a certain extent this will also affect the values of $R_{\text {cutoff }}$. They are not just the (energy independent) radii of the nuclei, but they rather describe the probability of nuclear interactions between the heavy ions. A more realistic calculation can be done by replacing this sharp cutoff with a smooth one, using the overlap function of Eq. (17) and (14). The $\Theta$-function in Eq. (28) has to be replaced by

$$
P(b)=1-\exp \left(-\sigma_{N N} T_{A B}(b)\right),
$$


with $\vec{b}=\vec{b}_{1}-\vec{b}_{2}$. Comparing this refined model with the one with a sharp cutoff, significant deviations are only present at the very upper end of the invariant mass range. Only the smallest impact parameter contribute significantly to these events, therefore a sensitivity to the cutoff is expected. All other (smaller) invariant masses are not very sensitive to the details of this cutoff.

Integrating over all $Y$ the cross section is

$$
\sigma_{c}=\int d W_{\gamma \gamma} \frac{d L}{d W_{\gamma \gamma}} \sigma_{\gamma \gamma}\left(W_{\gamma \gamma}\right) .
$$

For symmetric collisions $\left(R_{1}=R_{2}=R\right)$ we can write the luminosity in terms of a universal function $f(\tau)$ as

$$
d L / d W_{\gamma \gamma}=\frac{Z^{4} \alpha^{2}}{\pi^{4}} \frac{1}{W_{\gamma \gamma}} f(\tau),
$$

with $\tau=W_{\gamma \gamma} R / \gamma[14]$. For large values of $\gamma, f(\tau)$ is given by

$$
\begin{aligned}
f(\tau)= & \pi \tau^{2} \int_{1}^{\infty} u_{1} d u_{1} \int_{1}^{\infty} u_{2} d u_{2} \int_{0}^{2 \pi} d \phi \Theta\left(u_{1}^{2}+u_{2}^{2}-2 u_{1} u_{2} \cos \phi-4\right) \\
& \int_{-\infty}^{+\infty} d Y K_{1}^{2}\left(\frac{\tau}{2} u_{1} e^{Y}\right) K_{1}^{2}\left(\frac{\tau}{2} u_{2} e^{-Y}\right) .
\end{aligned}
$$

The function $f(\tau)$ is shown in Fig. 8, a useful parameterization of it was studied in [14].

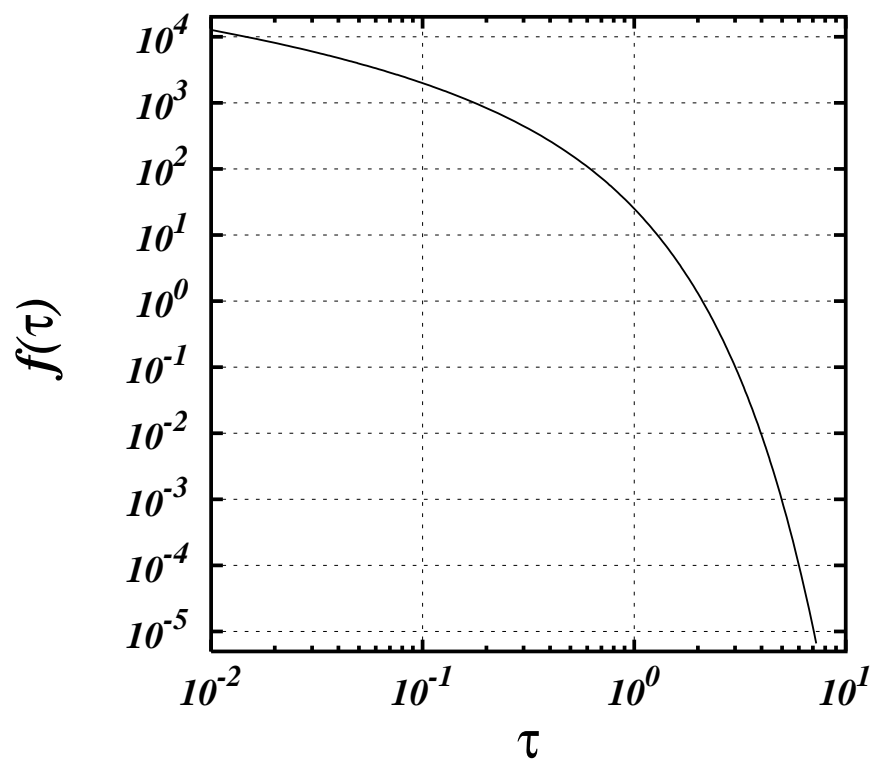

Figure 8: Plot of the universal function $f(\tau)$ (see Eq. (34)) as a function of $\tau=$ $W_{\gamma \gamma} R / \gamma$.

As a function of $Y$, the luminosity $d^{2} L / d W_{\gamma \gamma} d Y$ has a Gaussian shape with the maximum at $Y=0$. The width is approximately given by $\Delta Y=2 \ln \left[(2 \gamma) /\left(R W_{\gamma \gamma}\right)\right]$. Depending on the experimental situation additional cuts in the allowed $Y$ range are needed. 


\begin{tabular}{|c|c|c|c|c|}
\hline Ion & $Z, A$ & $\sqrt{s}$ & $\gamma$ & $L_{A A}\left(\mathrm{~cm}^{-2} \mathrm{~s}^{-1}\right)$ \\
\hline \multicolumn{5}{|c|}{$\mathrm{LHC}$} \\
\hline Pb-Pb & 82,208 & $1148 \mathrm{TeV}$ & 2950 & $1 \times 10^{26}$ \\
$\mathrm{Ca}-\mathrm{Ca}$ & 20,40 & $280 \mathrm{TeV}$ & 3750 & $4 \times 10^{30}$ \\
$p-p$ & 1,1 & $14 \mathrm{TeV}$ & 7450 & $10^{29}-10^{31}$ \\
\hline \multicolumn{5}{|c|}{$\mathrm{RHIC}$} \\
\hline $\mathrm{Au}-\mathrm{Au}$ & 79,197 & $20 \mathrm{TeV}$ & 106 & $2 \times 10^{26}$ \\
$\mathrm{I}-\mathrm{I}$ & 53,127 & $13 \mathrm{TeV}$ & 111 & $3 \times 10^{27}$ \\
$\mathrm{Cu}-\mathrm{Cu}$ & 29,63 & $7.2 \mathrm{TeV}$ & 122 & $1 \times 10^{28}$ \\
$\mathrm{Si}-\mathrm{Si}$ & 14,28 & $3.5 \mathrm{TeV}$ & 133 & $4 \times 10^{28}$ \\
$\mathrm{O}-\mathrm{O}$ & 8,16 & $2.0 \mathrm{TeV}$ & 133 & $1 \times 10^{29}$ \\
$p-p$ & 1,1 & $250 \mathrm{GeV}$ & 266 & $1 \times 10^{31}$ \\
\hline
\end{tabular}

Table 1: Parameters for different ion species at RHIC and LHC. In the entries we give the total invariant mass $\sqrt{s}$ of the system, the Lorentz factor $\gamma$ and the beam luminosity. Parameters are taken from [21, 23].

\subsection{Inelastic Vertices and Higher Order Corrections}

Heavy ions are complex objects unlike the pointlike, structureless electrons. The effects due to the finite size (and the nuclear interactions between them) has been considered by using an impact parameter approach. The additional processes coming from elastic nuclear interactions (diffractive processes, Pomeron interactions) will be considered separately in Sec. 4 and 6. Here we want to discuss mainly additional electromagnetic processes that can occur in distant collisions.

Especially for very heavy ions - like $\mathrm{Pb}-Z \alpha$ is no longer small. Therefore processes with more than one photon-exchange are important. In addition to the $\gamma \gamma$-process one can have electromagnetic dissociation of the ions. Furthermore the ions can also be excited due to the emission of the photon. This excitation can lead to an excited nucleus, or to the breakup of the nucleus, when the proton is knocked out of the nucleus due to the photon emission. At even higher $Q^{2}$ the photon can be emitted also from the quarks contained in the protons.

In Sec. 4 we will see that the electromagnetic dissociation of the ions is an important process. This cross section is often so large that it can also occur in addition to the $\gamma \gamma$-process.

In the impact parameter dependent approach the probability of several processes to occur in one collision can be calculated by assuming that the processes are independent of each other; their probabilities have to multiplied:

$$
P_{f A^{*}}(b)=P_{\gamma \gamma \rightarrow f}(b) P_{\gamma A \rightarrow A^{*}}(b) .
$$

Therefore the processes given in Fig. 9 are effectively included in this approach. This is a good approximation as long as the nucleus is not disturbed substantially. Integrating from $b=2 R$ up to infinity, one obtains the cross section for $\gamma \gamma \rightarrow f$ fusion accompanied by a specific $\gamma A \rightarrow A^{*}$ interaction

$$
\sigma_{f A^{*}}=2 \pi \int_{2 R}^{\infty} b d b P_{\gamma \gamma \rightarrow f}(b) P_{\gamma A \rightarrow A^{*}}(b) .
$$

Since $\sum_{A^{*}} P_{\gamma A \rightarrow A^{*}}=1$ (where we sum over all possible states $A^{*}$ of the nucleus including the ground state), we have

$$
\sum_{A^{*}} \sigma_{f A^{*}}=\sigma_{f}
$$



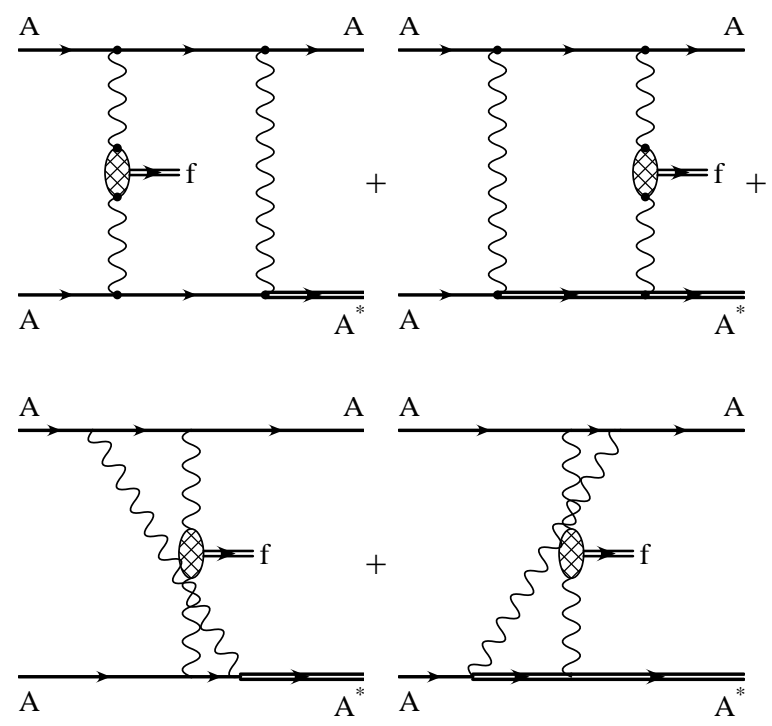

Figure 9: Due to the large charge of the ions, electromagnetic excitation in addition to the photon-photon process may occur.

that is, the same as the cross section of the $\gamma \gamma \rightarrow f$ process alone, without any higher order processes, as given above.

One can make an estimate of the importance of the higher order processes in the following way: The luminosity especially at high invariant masses peaks at $b \approx b_{\min }=2 R$ and one can therefore make the approximation

$$
\sigma_{f, A} \approx P_{A}(2 R) \sigma_{\gamma \gamma \rightarrow f},
$$

for the cross section $\sigma_{f, A}$, that is $\gamma \gamma \rightarrow f$ with the nucleus in the ground state in the final state and

$$
\sigma_{f, A^{*}} \approx\left(1-P_{A}(2 R)\right) \sigma_{\gamma \gamma \rightarrow f}
$$

for the nucleus to be excited. The impact parameter dependent probability for the excitation of the nucleus can be found in [1, 46 48]. They are strongly dependent on $A$. Especially important (see also Sec. 俰) is the giant dipole resonance (GDR), a highly coherent excited state, which can be interpreted as the movement of all neutrons against all protons. The probability of such an excitation of a nucleus with charge $Z_{2}$ and nucleon number $A_{2}\left(N_{2}=A_{2}-Z_{2}\right)$ by a nucleus with charge number $Z_{1}$ is given in [1] as

$$
P_{G D R}(b)=1-\exp \left(-S / b^{2}\right),
$$

with

$$
S=5.45 \times 10^{-5} \frac{Z_{2}^{2} N_{2} Z_{2}}{A_{2}^{2 / 3}} \mathrm{fm}^{2},
$$

where the Thomas-Reiche-Kuhn (TRK) sum rule was used and the position of the GDR is given by $80 A^{-1 / 3} \mathrm{MeV}$. For $\mathrm{Pb}-\mathrm{Pb}$ we get a value of $S=(10.4 \mathrm{fm})^{2}$. A parameterization which includes also all additional contributions (quasideuteron absorption, nucleon excitation, etc.) was given in 49]; they get a value of $S=$ $(17.4 \mathrm{fm})^{2}$. For the system Ca-Ca we get $S=(0.86 \mathrm{fm})^{2}$ using Eq. (41).

From this estimate one finds that about $75 \%$ of the photon-photon events in $\mathrm{Pb}-\mathrm{Pb}$ collisions are accompanied by an excitation, about $40 \%$ leading to the GDR. 
For Ca-Ca these are less than $2 \%$ of all events. As each of the ions can be excited, we get a total probability for excitation of at least one of them of about $2 \%$ for $\mathrm{Ca}-\mathrm{Ca}$ and of about $95 \%$ for $\mathrm{Pb}-\mathrm{Pb}$ (65\% from GDR). These effects are therefore dominant for $\mathrm{Pb}-\mathrm{Pb}$, but almost unimportant for $\mathrm{Ca}-\mathrm{Ca}$.

A detailed calculation of the cross section using the full $b$ dependence is shown in Fig. 10. It shows that the excitation probability is lower than the estimate given above.

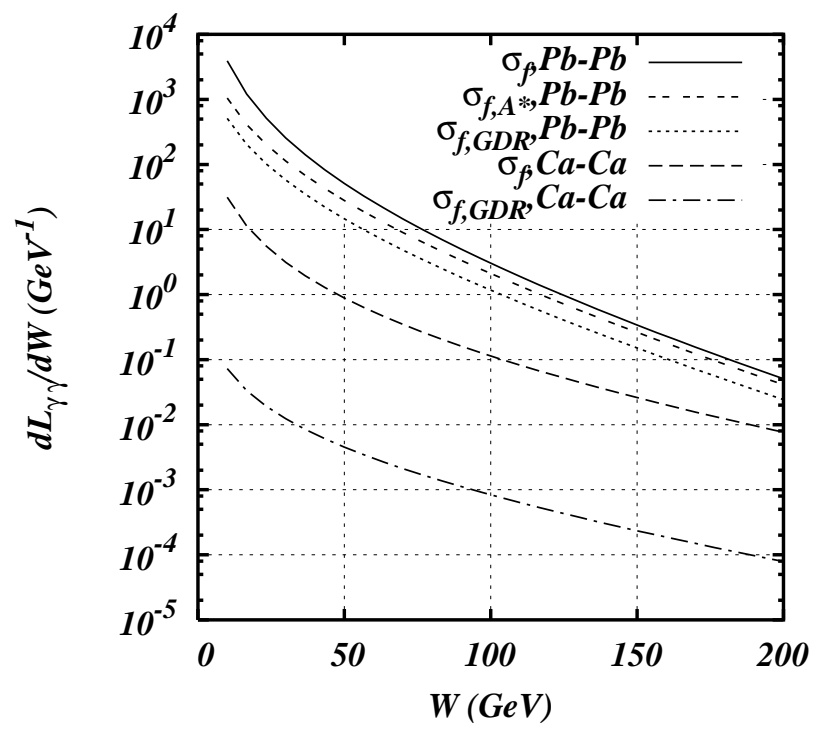

Figure 10: For $\mathrm{Pb}-\mathrm{Pb}$ collisions a substantial portion of the total luminosity ( $\sigma_{f}$, solid curve) comes from events with are accompanied by the excitation of at least one ion ( $\sigma_{A *}$, double-dashed curve) The dominant contribution is to the giant dipole resonance $\left(\sigma_{G D R}\right.$, dotted curve). For Ca-Ca collisions this is no longer very important. Shown are full calculation for the $\mathrm{LHC}(\gamma \approx 2950$ for $\mathrm{Pb}-\mathrm{Pb}, 3750$ for $\mathrm{Ca}-\mathrm{Ca}$ ).

The GDR excitation is followed most of the time by neutron evaporation. Also other photon induced reactions predominantly lead to the emission of individual nucleons. This emission of relatively low energy nucleons in the nucleus rest frame leads to high energetic neutrons (protons) with energies of about $3 \mathrm{TeV}$ (LHC) or 100 $\mathrm{GeV}$ (RHIC). The neutrons can possibly be detected in a zero degree calorimeter.

In 50] it was proposed to use the mutual emission of neutrons from both nuclei as a measure of the beam luminosity at RHIC. Using the coincidence of two neutrons in the very forward and backward direction other sources of neutrons can be suppressed effectively. Since the photonuclear processes are large and well understood they can lead to a good determination of the luminosity. The authors of [50] estimate to be able to determine the luminosity to about 5\%. It seems interesting to note that the $A$ dependence of the excitation cross section with two photons is given approximately by $10^{-9} A^{6} \mathrm{mb}[12$, the one-photon exchange cross section is given approximately by $10^{-5} A^{2} \mathrm{mb}$ [51], see Fig. 11. So for nuclei heavier than about C, the two-photon mechanism is dominant over the one-photon mechanism.

In the calculation of the luminosity we were always assuming that both nuclei remain in their ground state. But nuclei are weakly bound composite systems and it is possible that the photon emission leads to their excitation (see also Fig. 12). We distinguish two different types: those leading to an excited nucleus with a well defined excitation and the incoherent photon emission from individual protons 

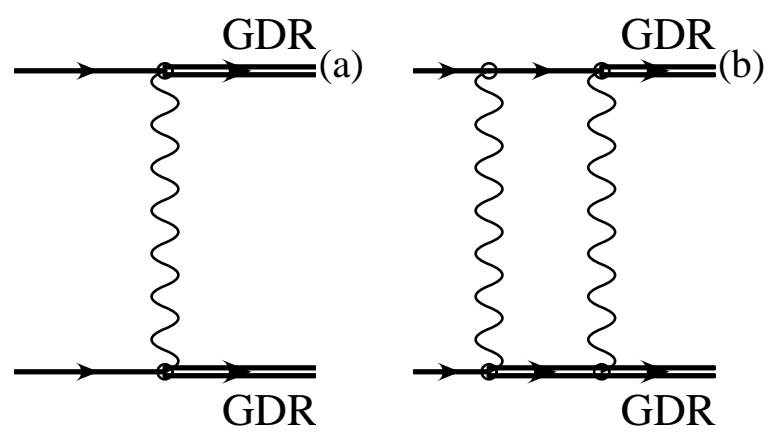

Figure 11: The mutual excitation process (a), where both ions are excited due to one-photon exchange, becomes less important at larger $A$ compared to the second order process (b).

(or even quarks within the protons), which are best treated as inclusive processes, summing over all excitation energies.

An equation for the inelastic photon-emission to a discrete state, was derived in [52] (see also 440]) using plane waves and therefore not subtracting the central collisions. It was applied to nuclear excitation, as well as to the case of the protonDelta transition [53]. The equivalent photon number can be expressed in terms of the structure functions $C$ and $D$ of the general hadron tensor

$$
\overline{\sum_{M_{i} M_{f}}} \Gamma^{\mu *} \Gamma^{\nu}=\left[g^{\mu \nu}-\frac{q^{\mu} q^{\nu}}{q^{2}}\right] C+\left[P^{\mu}-\frac{q P}{q^{2}} q^{\mu}\right]\left[P^{\nu}-\frac{q P}{q^{2}} q^{\nu}\right] D,
$$

where $\Gamma_{\mu}$ denotes the nuclear four-current. One obtains

$$
n(\omega)=\int \frac{\left(-2 C+q_{\perp} / \omega^{2} P^{2} D\right) \omega^{2}}{(2 \pi)^{3} 2 E P\left(q^{2}\right)^{2}} d^{2} q_{i}
$$

where $E$ and $P$ are energy and momentum of the nucleus. Whereas in the elastic case $q^{2}$ was given by $-\left(\omega^{2} / \gamma^{2}+q_{\perp}^{2}\right)$, it is here $\approx-\left[\frac{\omega}{\gamma}\left(\frac{\omega}{\gamma}+2 \Delta\right)\right]$, where $\Delta$ is the excitation energy. Using the Goldhaber-Teller model, the transition to the GDR was found to be very small, below $1 \%$ of the elastic contribution. Using the elastic structure functions of the proton in its usual dipole form (see, e.g., 40 the elastic proton equivalent photon number was obtained analytically in [54. Quite similarly the equivalent photon number corresponding to the $p-\Delta$ transition was obtained analytically in [53] using the structure functions of [55] as

$$
\begin{aligned}
n_{p \rightarrow \Delta}(\omega)= & \frac{\alpha}{4 \pi} \frac{\mu^{* 2}}{9 m^{2}}\left(\frac{m^{*}+m}{2 m}\right)^{2}\left[t _ { \operatorname { m i n } } \left\{\ln \left(\frac{t_{m i n}}{\Lambda^{2}+t_{\min }}\right)+\frac{11}{6}-\frac{2 t_{\min }}{\Lambda^{2}+t_{\min }}\right.\right. \\
& \left.\left.+\frac{3 t_{\min }^{2}}{2\left(\Lambda^{2}+t_{\min }\right)^{2}}-\frac{t_{\min }^{3}}{3\left(\Lambda^{2}+t_{\min }\right)^{3}}\right\}+\frac{\Lambda^{8}}{3\left(\Lambda^{2}+t_{\min }\right)^{3}}\right]
\end{aligned}
$$

with

$$
t_{\text {min }}=\frac{\omega^{2}}{\gamma^{2}}+\frac{\left(m^{*}-m\right)^{2}}{\gamma^{2}}+2 \frac{\omega\left(m^{*}-m\right)}{\gamma}
$$

and where $m^{*}=1232 \mathrm{MeV}$ is the mass of the $\Delta, m=938 \mathrm{MeV}$ the proton mass, $\Lambda^{2}=0.71 \mathrm{GeV}^{2}$ and $\mu^{*}=9.42$. For not too large photon energy $\omega$ it is given by a 
constant

$$
n_{\Delta}(\omega) \approx \frac{\alpha}{4 \pi} \frac{\mu^{* 2}}{9 m^{2}}\left(\frac{m^{*}+m}{2 m}\right)^{4} \frac{\Lambda^{2}}{3} .
$$

This is an effect of the order of $10 \%$ [53.
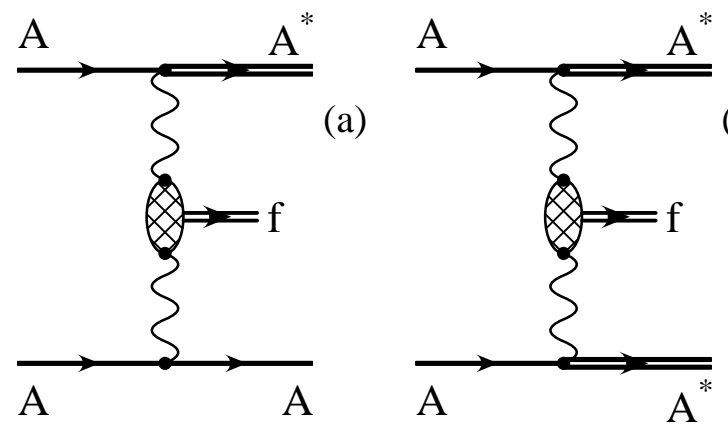

(b)

Figure 12: In photon-photon collisions, one (Fig. (a)) and even both (Fig. (b)) nuclear vertices can be inelastic, leading to excited nuclei.

As we have seen above, the knowledge of impact parameter dependent equivalent photon numbers is very helpful, as it allows in a direct way to take the strong absorption into account (Eq. (28)). The equivalent photon spectrum corresponding to a fast moving point particle is given by Eq. 222). This result can now be generalized to arbitrary charge-current distributions (also quantum-mechanical transition currents in the framework of the Glauber theory).

The equivalent photon spectrum corresponding to a spherically symmetric charge distribution $\rho(r)$ moving with velocity $v$ at an impact parameter $b$ is derived in [43. The Fourier transformation of this charge distribution, with $\int d^{3} r \rho(r)=Z$, is given by

$$
f\left(k^{2}\right)=Z F\left(k^{2}\right)=\int d^{3} r \exp (-i \vec{k} \vec{r}) \rho(r) .
$$

For $\gamma \gg 1$ we only need the perpendicular component of the electric and magnetic fields, $\vec{E}_{\perp}$ and $\vec{B}_{\perp}$. One finds 43

$$
\vec{E}_{\perp}=\int \frac{d^{2} k_{\perp}}{(2 \pi)^{2}} \frac{Z e}{v k^{2}} f\left(k^{2}\right) \exp \left(-i \vec{k}_{\perp} \vec{b}\right) \vec{k}_{\perp},
$$

where $k^{2} \approx-k_{\perp}^{2}-(\omega / \gamma)^{2}$. It can easily be seen, that $E_{\perp} \| \vec{b}$ and one obtains the equivalent photon number as

$$
N(\omega, b)=\frac{Z^{2} \alpha}{\pi^{2}}\left(\frac{c}{v}\right)^{2} \frac{1}{b^{2}}\left|\int_{0}^{\infty} d u u^{2} J_{1}(u) \frac{f\left(-\frac{x^{2}+u^{2}}{b^{2}}\right)}{x^{2}+u^{2}}\right|^{2}
$$

where $x=\omega b / \gamma$.

In a similar way the equivalent photon spectrum of a point-like magnetic dipole, moving with a constant velocity at a given impact parameter, is calculated in [56]. This purely classical result can also be interpreted quantum-mechanically by using the corresponding electromagnetic matrix-elements.

An interesting question is the incoherent contributions due to the protons inside the nucleus. We generalize Eqs. (47)-(49) in the following way: The static 
charge distribution is replaced by a transition charge density $\rho_{f 0}(r)$ and the Fourier transform of it is given by

$$
f_{f 0}(\vec{k})=\int d^{3} r \exp (-i \vec{k} \vec{r}) \rho_{f 0}(r)
$$

where $f$ is some final state of the nucleus and

$$
\begin{aligned}
f_{f 0}(\vec{k}) & =\int d^{3} r d \xi \Psi_{f}^{*}(\xi) \sum_{i=1}^{Z} \delta\left(\vec{r}-\vec{r}_{i}\right) \Psi_{0}(\xi) \exp (-i \vec{k} \vec{r}) \\
& =\sum_{i=1}^{Z} \int d \xi \Psi_{f}^{*}(\xi) \exp \left(-i \vec{k} \vec{r}_{i}\right) \Psi_{0}(\xi)
\end{aligned}
$$

where $\xi=r_{1}, r_{2}, \cdots, r_{Z}$ is the set of all proton coordinates (for our argument we can neglect the neutrons). We get the total incoherent contribution by summing over all states $f$ excluding the ground state $f=0$. The sum can be performed using the closure relation and we obtain:

$$
\begin{aligned}
S\left(\vec{k}, \vec{k}^{\prime}\right)= & \sum_{f \neq 0} f_{f 0}^{*}(\vec{k}) f_{f 0}\left(\vec{k}^{\prime}\right) \\
= & \int d \xi d \xi^{\prime} \sum_{i, j} \Psi_{0}^{*}(\xi) \exp \left(i \vec{k} \vec{r}_{i}\right) \Psi_{f}(\xi) \Psi_{f}^{*}\left(\xi^{\prime}\right) \exp \left(-i \vec{k}^{\prime} \vec{r}_{j}^{\prime}\right) \Psi_{0}\left(\xi^{\prime}\right) \\
& -f_{00}^{*}(\vec{k}) f_{00}\left(\vec{k}^{\prime}\right) \\
= & \sum_{i, j} \int d \xi\left|\Psi_{0}(\xi)\right|^{2} \exp \left(i \vec{k} \vec{r}_{i}-i \vec{k}^{\prime} \vec{r}_{j}\right)-f_{00}^{*}(\vec{k}) f_{00}\left(\vec{k}^{\prime}\right) .
\end{aligned}
$$

We split the sum over $i, j$ now into one for $i=j$ and one for $i \neq j$, following [57]. In the limit of no correlation (not even Pauli correlations) we obtain

$$
S\left(\vec{k}, \vec{k}^{\prime}\right)=Z F\left(\vec{k}-\vec{k}^{\prime}\right)-Z F(\vec{k}) F\left(\vec{k}^{\prime}\right),
$$

where we have introduced a normalized form factor $F(\vec{k})$ of the nucleus as $F(\vec{k})=$ $f_{00}(\vec{k}) / Z$, i.e., $F(0)=1$.

The equivalent photon number due to the incoherent contribution can now be written as

$$
N^{i n c o h}(\omega, b)=\frac{\alpha}{\pi^{2}}\left(\frac{c}{v}\right)^{2} \frac{1}{b^{2}} \iint d^{2} k_{\perp} d^{2} k_{\perp}^{\prime} \vec{k}_{\perp} \vec{k}_{\perp}^{\prime} \frac{\exp \left(i \overrightarrow{k_{\perp}} \vec{b}-i \vec{k}_{\perp}^{\prime} \vec{b}\right)}{k^{2} k^{\prime 2}} S\left(\vec{k}, \vec{k}^{\prime}\right),
$$

where $\vec{k}=\left(\vec{k}_{\perp}, \omega / v\right), \vec{k}^{\prime}=\left(\vec{k}_{\perp}^{\prime}, \omega / v\right)$. Using Eqs. (47) and (54) and defining a thickness function $T_{z}(b)$ - see Eq. (13) - where the nucleon density $n_{A}$ is now replaced by the charge density $\rho$ ), we have

$$
N^{\text {incoh }}(\omega, b)=\int d^{2} r_{\perp} T_{z}\left(r_{\perp}\right) N^{\text {point }}\left(\omega, \vec{b}+\vec{r}_{\perp}\right)-Z N^{\text {form }}(\omega, b),
$$

where $N^{\text {form }}$ is the usual equivalent photon number for a given form factor (divided by $Z^{2}$ ), see Eq. (49), $N^{\text {point }}$ denotes the equivalent photon spectrum due to a point charge. For $b=0$ this expression would diverge, and a suitable cut-off has to be introduced $\left(b_{\min }=R_{\text {proton }}\right.$, or another value of $b_{\text {min }}$, for which the equivalent photon approximation ceases to be valid). If one is integrating over all impact parameters (therefore also including central collisions), the total incoherent equivalent photon number can be defined:

$$
n^{\text {incoh }}(\omega)=Z\left[n^{\text {point }}(\omega)-n^{\text {form }}(\omega)\right],
$$


where $n^{i}(\omega)=\int d^{2} b N^{i}(\omega, b)$ and we have used $\int d^{2} r_{\perp} T_{z}\left(\vec{r}_{\perp}\right)=Z$.

Incoherent scattering is a well known general phenomenon. E.g., the blue sky is caused by the incoherent scattering of sunlight by gas molecules or other randomly distributed dipole scatterer (see, e.g., p.418ff of [30]). It can also be formulated in a way that makes a connection with the parton model. As an example let us look at the contribution from incoherent photon emissions of the quarks in the proton. In the parton model a proton in the infinite momentum frame consists of partons (quarks, gluons,...). We use the plane wave approach (see 40]), where the impact parameter dependence is not manifest. The equivalent photon spectrum corresponding to a given proton final state can be expressed in terms of the structure functions $W_{1}$ and $W_{2}$. We only consider the dominant term corresponding to $W_{2}$. We are also interested only in inclusive reactions, therefore we integrate over the invariant mass of the final state $M^{2}$. One has [40, 58]

$$
n(\omega)=\frac{\alpha}{\pi} \iint \frac{d Q^{2} d M^{2}}{\left(Q^{2}\right)^{2}}\left|q_{\perp}\right|^{2} \frac{1}{2 m} W_{2}\left(M^{2}, Q^{2}\right),
$$

with $Q^{2}=Q_{m i n}^{2}(\omega)+q_{\perp}^{2}$. Since $p=p^{\prime}+q$ we obtain

$$
M^{2}=m^{2}+2 m \nu-Q^{2},
$$

with $\nu=-p q / m$. (Since we assume that the photon is emitted, not absorbed, from the nuclear system, our sign of $q$ and $\nu$ is somewhat unconventional.) Introducing the scaling variable $x=Q^{2} /(2 m \nu)$, we can write in the scaling limit, see e.g. [58],

$$
W_{2}\left(M^{2}, Q^{2}\right)=\frac{1}{\nu} F_{2}(x) .
$$

Changing the integration variable $d M^{2}$ to $d x$, we obtain

$$
n(\omega)=\frac{\alpha}{\pi} \int d x F_{2}(x) \frac{1}{x} \int d Q^{2} \frac{\left|q_{\perp}\right|^{2}}{\left(Q^{2}\right)^{2}} .
$$

We express the structure function $F_{2}(x)$ in terms of the quark distribution functions $f_{q_{i} \mid p}(x)$ :

$$
F_{2}(x)=x \sum_{q_{i}} e_{i}^{2} f_{q_{i} \mid p}(x),
$$

where $e_{i}$ is the charge of the quark $q_{i}(i=u, d, s, \cdots)$. Eq. (61) now has an intuitive interpretation: The proton consist of partons (=quarks) with an momentum fraction $x$, they radiate as pointlike particles. This is described by the $d Q^{2}$ integration. Taking also the energy loss of the quarks into account in a more complete formula, the photon spectrum from a quark is given by

$$
f_{\gamma / q}(z)=\frac{\alpha}{2 \pi} \frac{1+(1-z)^{2}}{z} \log \left(\frac{Q_{1}^{2}}{Q_{2}^{2}}\right),
$$

where $z$ is the ratio of photon-energy $\omega$ and energy of the quark $x E$.

According to [59] we choose $Q_{1}^{2}$ to be the maximum value of the momentum transfer given by $x_{1} x_{2} z_{1} z_{2} s / 4-m^{2}$ and the choice of the minimum $Q_{2}^{2}=1 \mathrm{GeV}^{2}$ is made such that the photons are sufficiently off shell for the quark-parton model to be applicable. The inelastic contribution to the $\gamma \gamma$-cross section is then given by

$$
\begin{aligned}
\sigma_{p p}^{i n e l}= & \sum_{i j} e_{i}^{2} e_{j}^{2} \int_{\frac{4 m^{2}}{s}}^{1} d x_{1} \int_{\frac{4 m^{2}}{s x_{1}}}^{1} d x_{2} \int_{\frac{4 m^{2}}{s x_{2}}}^{1} d z_{1} \int_{\frac{4 m^{2}}{s x_{1} x_{1}}}^{1} d z_{2} \\
& f_{q_{i} \mid p}\left(x_{1}, Q^{2}\right) f_{q_{j} \mid p}\left(x_{1}, Q^{2}\right) f_{\gamma / q_{i}}\left(z_{1}\right) f_{\gamma / q_{j}}\left(z_{2}\right) \\
& \hat{\sigma}_{\gamma \gamma}\left(W_{\gamma \gamma}=\sqrt{x_{1} x_{2} z_{1} z_{2} s / 4}\right)
\end{aligned}
$$


(Eq. (3) of [59]) and where $s$ is the invariant mass of the $p p$ system. Of course the photons are now somewhat more off-shell than in the elastic case, and for some cases it could be less safe to use the assumption of real photons $\left(q^{2}=0\right)$, when calculating the cross section for the $\gamma \gamma$-subprocess $\hat{\sigma}$.

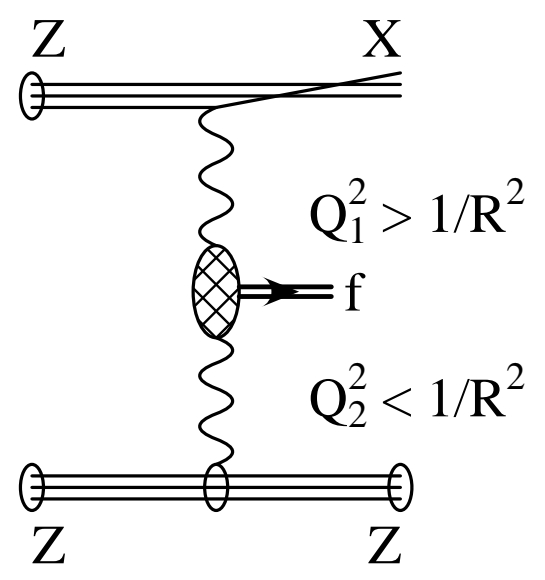

Figure 13: With $Q^{2}<1 / R^{2}$ the photon is emitted coherently from all "partons" inside the ion. For $Q^{2} \gg 1 / R^{2}$ the "partonic" structure of the ion is resolved.

A similar formula can also be written for the semielastic ( = elastic-inelastic) cross section, see [59,60]. $\gamma \gamma$-luminosities are calculated according to this procedure in both of these papers. The MRSD' parameterization [61, 62] for the partonic densities is used in [59], in [60] the simple parameterization $F_{2}=0.16 \ln (1 / x)$ was used. One finds that typically the inel-inel contribution is largest, as the charges of the partons (quarks) are comparable to the charges of the proton.

For the proton contribution to the photon spectrum of a heavy nucleus, the situation is different. The coherent contribution is proportional to $Z^{2}$, whereas they are only proportional to $Z$ for the incoherent one. As $Z \gg 1$ one expects the coherent part to be dominant.

\section{$4 \gamma$-A interactions}

The cross section for the collisions of the equivalent photons of one nucleus with the other is given by (see Eq. (25)):

$$
\sigma=\int \frac{d \omega}{\omega} n(\omega) \sigma_{\gamma}(\omega)
$$

where the equivalent photon number $n(\omega)$ is given in Eq. (24) and $\sigma_{\gamma}(\omega)$ is the photonuclear cross section. This gives rise to many interesting phenomena ranging from the excitation of discrete nuclear states, giant multipole resonances (especially the giant dipole resonance), quasideuteron absorption, nucleon resonance excitation to the nucleon continuum (see, e.g.. [33, 63]). Photo-induced processes lead in general to a change of the charge-to-mass ratio of the nuclei, and with their large cross section they are therefore a serious source of beam loss. Especially the cross section for the excitation of the the giant dipole resonance, a collective mode of the nucleus, is rather large for the heavy systems (of the order of 100b). For a recent discussion see 64. The cross section scales approximately with $Z^{10 / 3}$. (Another 
serious source of beam loss, the $e^{+} e^{-}$bound-free pair creation will be discussed in Sec. 7 ). The contribution of the nucleon resonances (especially the $\Delta$ resonance) has also been confirmed experimentally in fixed target experiments with 60 and 200 $\mathrm{GeV} / \mathrm{A}$ (heavy ions at CERN, "electromagnetic spallation") [65 68]. For details of these aspects, we refer the reader to [16, 47, 48, 69], where scaling laws, as well as detailed calculations for individual cases are given.

Recently the total dissociation cross section for different ion species was studied in an experiment at CERN 70. There it was found that this cross section is dominated at medium and large $Z$ by the electromagnetic dissociation, with the region of the GDR contributing with about $80 \%$. The theoretical calculations, which can be considered to be fairly reliable and detailed, see e.g. [71, agree quite well with the experiments apart from an additional effect, which can be parameterized as $\sigma_{a d d}=0.12 Z$ barn, a very sizeable effect (For large $Z$ it is even larger than the nuclear cross section). It is tempting to guess that the $Z$ dependence is due to an incoherent effect of the $Z$ protons in a nucleus. However the corresponding incoherent photon number (Eq. (56)) is very small for the relevant region $b>$ $R_{1}+R_{2}$. Therefore we exclude an incoherent effect as the explanation of the anomaly observed in [70].

The interaction of quasireal photons with protons has been studied extensively at the electron-proton collider HERA (DESY, Hamburg), with $\sqrt{s}=300 \mathrm{GeV}$ $\left(E_{e}=27.5 \mathrm{GeV}\right.$ and $E_{p}=820 \mathrm{GeV}$ in the laboratory system). This is made possible by the large flux of quasi-real photons from the electron (positron) beam (for a review see [38]). The obtained $\gamma p$ center-of-mass energies (up to $W_{\gamma p} \approx$ $200 \mathrm{GeV}$ ) are an order of magnitude larger than those reached by fixed target experiments. Similar and more detailed studies will be possible at the relativistic heavy ion colliders RHIC and LHC, due to the larger flux of quasireal photons from one of the colliding nuclei. In the photon-nucleon subsystem, one can reach invariant masses $W_{\gamma N}$ up to $W_{\gamma N, \max }=\sqrt{4 W_{\max } E_{N}} \approx 0.8 \gamma A^{-1 / 6} \mathrm{GeV}$. In the case of RHIC ( $\left.{ }^{197} \mathrm{Au}, \gamma=106\right)$ this is about $30 \mathrm{GeV}$, for LHC $\left({ }^{208} \mathrm{~Pb}, \gamma=2950\right)$ one obtains $950 \mathrm{GeV}$. Thus one can study physics quite similar to the one at HERA, with nuclei instead of protons. Photon-nucleon physics includes many aspects, like the energy dependence of total cross-sections, diffractive and non-diffractive processes (see, e.g., 38]). An important subject is elastic vector meson production $\gamma p \rightarrow V p$ (with $V=\rho, \omega, \phi, J / \Psi, \ldots$ ). A review of exclusive neutral vector meson production is given in [72]. The diffractive production of vector mesons allows one to get insight into the interface between perturbative QCD and hadronic physics. Elastic processes (i.e., the proton remains in the ground state) have to be described within nonperturbative (and therefore phenomenological) models. It was shown in 73 that diffractive ("elastic") $J / \Psi$ photoproduction is a probe of the gluon density at $x \approx \frac{M_{\Psi}^{2}}{W_{\gamma N}^{2}}$ (for quasireal photons). Inelastic $J / \Psi$ photoproduction was also studied recently at HERA [74. Going to the hard exclusive photoproduction of heavy mesons on the other hand, perturbative QCD is applicable. Recent data from HERA on the photoproduction of $J / \Psi$ mesons have shown a rapid increase of the total cross section with $W_{\gamma N}$, as predicted by perturbative QCD. Such studies could be extended to photon-nucleus interactions at RHIC, thus complementing the HERA studies. Equivalent photon flux factors are large for the heavy ions due to coherence. On the other hand, the A-A luminosities are quite low, as compared to HERA. Of special interest is the coupling of the photon of one nucleus to the Pomeron-field of the other nucleus. Such studies are envisaged for RHIC, see [18 21] where also experimental feasibility studies were performed.

It is useful to have estimates of the order of magnitude of vector meson production in photon-nucleon processes at RHIC and LHC. Let us assume a cross-section 
that rises with the $\gamma p$ center of mass energy approximately with a power law:

$$
\sigma_{\gamma}=\sigma_{0}\left(\frac{W_{\gamma N}}{W_{0}}\right)^{\beta}
$$

with $W_{0}$ chosen to be $1 \mathrm{GeV}$ and $\beta \approx 0.22$ for $V=\rho, \omega, \phi$, and $\beta \approx 0.8$ for $V=J / \Psi$. Also the total hadronic interaction cross section can be parameterized in this form with $\beta \approx 0.16$. From Fig. 17 of [38] or Fig. 5 of [75] one has for $\sigma_{0} \approx 50 \mu$ b for the total hadronic cross section, $5 \mu \mathrm{b}$ for $V=\rho, 0.5 \mu \mathrm{b}$ for $V=\omega, \phi$ and $10^{-3} \mu \mathrm{b}$ for $V=J / \Psi$. Making use of the photon number of Eq. (26) the total cross section for vector meson production on the reaction $Z+p \rightarrow Z+p+V$ due to the equivalent photon spectrum of the nucleus $Z$ is obtained as

$$
\begin{aligned}
\sigma= & \frac{2 Z^{2} \alpha}{\pi} \sigma_{0}\left(\frac{2 m_{N} \gamma_{p}}{R W_{0}^{2}}\right)^{\beta / 2} \times \\
& {\left[\left(1-\left(\frac{\omega_{\min } R}{\gamma_{p}}\right)^{\beta / 2}\right) \frac{4}{\beta^{2}}+\left(\frac{\omega_{\min } R}{\gamma_{p}}\right)^{\beta / 2} \frac{2 \ln \left(\omega_{\min } R / \gamma_{p}\right)}{\beta}\right], }
\end{aligned}
$$

where some minimum value of the energy of the equivalent photon is used, say $\omega_{\text {min }}=1 \mathrm{GeV}$ for the total hadronic cross section, $\omega_{\text {min }}=2 \mathrm{GeV}$ for $V=\rho, \omega$ and $\phi$ and $\omega_{\min }=10 \mathrm{GeV}$ for $V=J / \Psi$, and $\omega_{\max }=\gamma_{p} / R$. For $\beta=0$ one obtains in a similar way

$$
\sigma=\frac{Z^{2} \alpha}{\pi} \sigma_{0}\left[\ln \left(\frac{\gamma_{p}}{\omega_{\min } R}\right)\right]^{2} .
$$

The Lorentz factor $\gamma_{p}$ of the nucleus $Z$, as viewed from the proton, is given by

$$
\gamma_{p}=2 \gamma^{2}-1 \text {. }
$$

We assume that a proton and a nucleus $Z$ collide, with the same value $\gamma$ (see Fig. 1, where one nucleus is replaced by a proton). We obtain the following numbers, shown in Table 2 .

\begin{tabular}{|c|c|c|c|c|}
\hline & \multicolumn{2}{|c|}{ RHIC } & \multicolumn{2}{c|}{ LHC } \\
\hline Ion & I & $\mathrm{Au}$ & $\mathrm{Ca}$ & $\mathrm{Pb}$ \\
$\gamma$ & 111 & 106 & 3750 & 2950 \\
$R(\mathrm{fm})$ & 6 & 7 & 4 & 7 \\
\hline$\sigma_{\text {tot }}(\mathrm{mb})$ & 20 & 40 & 15 & 200 \\
$\sigma_{\rho}(\mathrm{mb})$ & 2 & 3.5 & 1.5 & 25 \\
$\sigma_{\omega}, \sigma_{\phi}(\mathrm{mb})$ & 0.2 & 0.35 & 0.15 & 2.5 \\
$\sigma_{J / \Psi}(\mu \mathrm{b})$ & 0.5 & 1.5 & 3 & 40 \\
\hline
\end{tabular}

Table 2: The expected cross sections for the elastic vector meson production on a proton induced by the equivalent photons of a nucleus $Z$ are given for RHIC and LHC conditions. Also shown are the total electromagnetic cross sections $\sigma_{\text {tot }}$. See text for details.

The numbers in Table 2 refer to the photoproduction on one proton. In $A A$ collisions there is incoherent photoproduction on the individual $A$ nucleons. Shadowing effects will occur in the nuclear environment and it will be interesting to study these. There is also the coherent contribution where the nucleus remains in the ground state. Due to the large momentum transfer, the total (angle integrated) coherent scattering shows an undramatic $A^{4 / 3}$ dependence. This is in contrast to, 
e.g., low energy $\nu \mathrm{A}$ elastic scattering, where the coherence effect leads to an $A^{2}$ dependence, which is relevant for the stellar collapse, see, e.g., [76], where also a pedagogical general discussion of coherence effects is given. In addition there are inelastic contributions, where the proton (nucleon) is transformed into some final state $X$ during the interaction (see $[74]$ ). The experimental possibilities of this at RHIC are investigated in [18, 20, 21].

At the LHC one can extend these processes to much higher invariant masses $W$, therefore much smaller values of $x$ will be probed. Whereas the $J / \Psi$ production at HERA was measured up to invariant masses of $W \approx 160 \mathrm{GeV}$, the energies at the LHC allow for studies up to $\approx 1 \mathrm{TeV}$.

At the FELIX detector at LHC 23] hard diffractive vector meson photoproduction can be investigated especially well in $A A$ collisions. In comparison to previous experiments, the very large photon luminosity should allow observation of processes with quite small $\gamma p$ cross sections, such as $\Upsilon$-production. For more details see 23.

$C=-1$ vector mesons can be produced in principle by the fusion of three (or, less important, five, seven, ... ) equivalent photons (see Fig. 14). The cross section scales with $Z^{6}$. This graph has been calculated by I. Ginzburg et al. [77] using the methods of $[78$ 80]. It is smaller than the contributions discussed above, even for nuclei with large $Z$. Quite similarly the QED analogue of the $C=-1$ mesons, the ortho-states of positronium, muonium, or tauonium can be calculated 81.

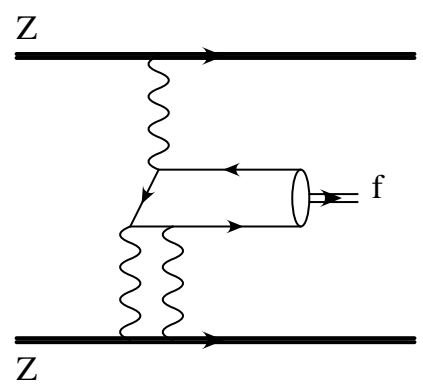

Figure 14: By using three (and more) photon processes also states that are forbidden in the two-photon fusion process can be produced. Interesting final states $f$ are, for example, orthopositronium, orthomuonium, or the vector mesons $\rho, \omega, \phi$.

One might have thought that these three- and more photon contributions are of the same order of magnitude, since $Z \alpha \leq 1$. But there is another scale: As one can see from Eq. (76) below, there is another factor $1 /(m b)$. For electrons the important range $b \approx 1 / m$ and this factor therefore is of the order of unity. From this one expects that orthopositronium production can be similar in size than parapositronium production. For heavier systems the impact parameter range $b$ cannot be smaller than $R$, the nuclear radius. Putting $1 / R=\Lambda \approx 30 \mathrm{MeV}$ (for $R \approx$ $7 \mathrm{fm}$ ), the scale factor is $\Lambda / M$ with $M$ the mass of the produced particle $M=m_{\mu}$, .... This factor is always (much) smaller than one, thus the production via these higher order processes is small. Of course the above arguments are rather qualitative and should be complemented by more detailed calculations. Such calculations can be done using the technique developed for vector meson production in proton-proton collisions via gluon-exchange processes [78 80 .

As another possibility we mention photon-gluon fusion leading to the production of $c \bar{c}$ and $b \bar{b}$ quark pairs. It was suggested in Ref. [82] as a possibility to deduce the in-medium gluon distribution. Further studies were done in Refs. [83, 84], and this possibility is reviewed in [16]. 


\section{Photon-Photon Physics at various invariant mass scales}

Scattering of light on light, while absent in classical Maxwell electrodynamics, takes place due to quantum effects, like pair creation. At low energies, photon-photon scattering is dominated by electron intermediate states, the scattering of light on light occurs in higher orders via an electron loop, see, e.g., [85. The lowest order process is $e^{+} e^{-}$pair creation and is well described by QED.

Up to now photon-photon scattering has been mainly studied at $e^{+} e^{-}$colliders. Many reviews [40, 86, 87] as well as conference reports [88, 91] exist. The traditional range of invariant masses has been the region of mesons, ranging from $\pi^{0}\left(m_{\pi^{0}}=\right.$ $135 \mathrm{MeV})$ up to about $\eta_{c}\left(m_{\eta_{c}}=2980 \mathrm{MeV}\right)$. Recently the total $\gamma \gamma \rightarrow$ hadron crosssection has been studied at LEP2 up to an invariant mass range of about $70 \mathrm{GeV}$ [92]. We are concerned here mainly with the invariant mass region relevant for RHIC and LHC (see the $\gamma \gamma$-luminosity figures below). Apart from the production of $e^{+} e^{-}$(and $\mu^{+} \mu^{-}$) pairs, the photons can always be considered as quasireal. The cross section section for virtual photons deviates from the one for real photons only for $Q^{2}$, which are much larger then the coherence limit $Q^{2} \lesssim 1 / R^{2}$ (see also the discussion in 40]). For real photons general symmetry requirements restrict the possible final states, as is well known from the Landau-Yang theorem 93. Especially it is impossible to produce spin 1 final states. In $e^{+} e^{-}$annihilation only states with $J^{P C}=1^{--}$can be produced directly. Two photon collisions give access to most of the $C=+1$ mesons.

The cross section for $\gamma \gamma$-production in a heavy ion collision factorizes into a $\gamma \gamma$ luminosity function and a cross-section $\sigma_{\gamma \gamma}\left(W_{\gamma \gamma}\right)$ for the reaction of the (quasi)real photons $\gamma \gamma \rightarrow f$, where $f$ is any final state of interest (see Eq. (27). When the final state is a narrow resonance, the cross-section for its production in two-photon collisions is given by

$$
\sigma_{\gamma \gamma \rightarrow R}\left(M^{2}\right)=8 \pi^{2}\left(2 J_{R}+1\right) \Gamma_{\gamma \gamma}(R) \delta\left(M^{2}-M_{R}^{2}\right) / M_{R},
$$

where $J_{R}, M_{R}$ and $\Gamma_{\gamma \gamma}(R)$ are the spin, mass and two-photon width of the resonance $R$. This makes it easy to calculate the production cross-section $\sigma_{A A \rightarrow A A+R}$ of a particle in terms of its basic properties. In Fig. 15 the function $4 \pi^{2} d L_{\gamma \gamma} / d M / M^{2}$ is plotted for various systems. It can be directly used to calculate the cross-section for the production of a resonance $R$ with the formula

$$
\sigma_{A A \rightarrow A A+R}(M)=\left(2 J_{R}+1\right) \Gamma_{\gamma \gamma} \frac{4 \pi^{2} d L_{\gamma \gamma} / d M}{M^{2}} .
$$

We will now give a general discussion of possible photon-photon physics at relativistic heavy ion colliders. Invariant masses up to several $\mathrm{GeV}$ can be reached at RHIC and up to about $100 \mathrm{GeV}$ at LHC.

We can divide our discussion into the following two main subsections: Basic QCD phenomena in $\gamma \gamma$-collisions (covering the range of meson, meson-pair production, etc.) and $\gamma \gamma$-collisions as a tool for new physics, especially at very high invariant masses.

\subsection{Basic QCD phenomena in $\gamma \gamma$-collisions}

\subsubsection{Hadron spectroscopy: Light quark spectroscopy}

One may say that photon-photon collisions provide an independent view of the meson and baryon spectroscopy. They provide powerful information on both the flavor and spin/angular momentum internal structure of the mesons. Much has 

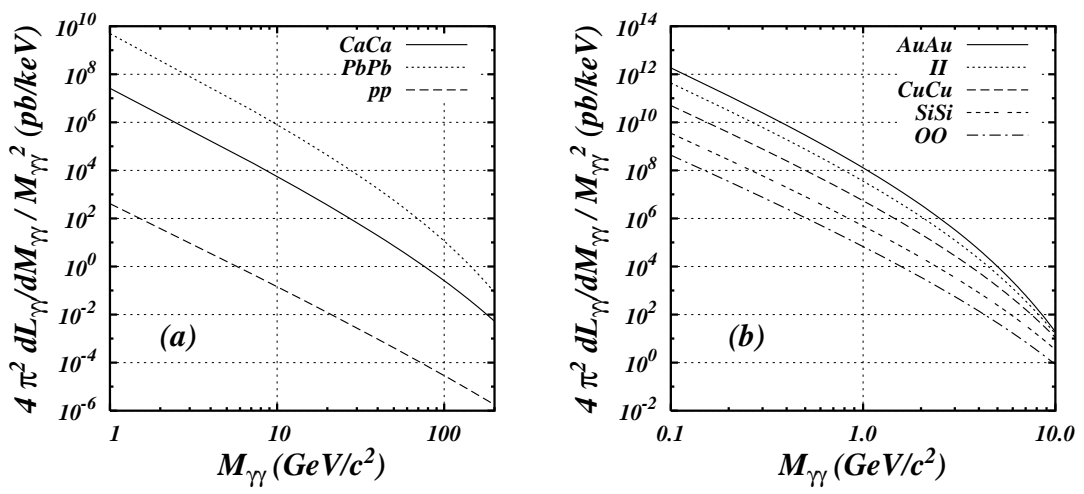

Figure 15: The universal function $4 \pi^{2} d L_{\gamma \gamma} / d M_{\gamma \gamma} / M_{\gamma \gamma}^{2}$ is plotted for different ion species at LHC (a) and RHIC (b). For the parameters used see table $\mathrm{Q}$.

already been done at $e^{+} e^{-}$colliders. For a review see, e.g., 94. Light quark spectroscopy is very well possible at RHIC, benefiting from the high $\gamma \gamma$-luminosities. Detailed feasibility studies exist [18 21]. In this study, $\gamma \gamma$ signals and backgrounds from grazing nuclear and beam gas collisions were simulated with both the FRITIOF and VENUS Monte Carlo codes. The narrow $p_{\perp}$-spectra of the $\gamma \gamma$-signals provide a good discrimination against the background. The possibilities of the LHC are given in the FELIX LoI [23].

The absence of meson production via $\gamma \gamma$-fusion is also of great interest for glueball search. The two-photon width of a resonance is a probe of the charge of its constituents, so the magnitude of the two-photon coupling can serve to distinguish quark dominated resonances from glue-dominated resonances ("glueballs"). In $\gamma \gamma$ collisions, a glueball can only be produced via the annihilation of a $q \bar{q}$ pair into a pair of gluons, whereas a normal $q \bar{q}$-meson can be produced directly, so we estimate

$$
\frac{\sigma(\gamma \gamma \rightarrow M)}{\sigma(\gamma \gamma \rightarrow G)}=\frac{\Gamma(M \rightarrow \gamma \gamma)}{\Gamma(G \rightarrow \gamma \gamma)} \sim \frac{1}{\alpha_{s}^{2}},
$$

where $\alpha_{s}$ is the strong interaction coupling constant and where $G$ is a "glueball", $M$ a normal $q \bar{q}$-meson. Glueballs are produced most easily in gluon-rich environment. This is, e.g., the case in radiative $J / \Psi$ decays, $J / \Psi \rightarrow \gamma g g$.

In order to form a meson out of the gluon pair, they must first annihilate into a $q \bar{q}$ pair. So we estimate

$$
\frac{\Gamma(J / \Psi \rightarrow \gamma G)}{\Gamma(J / \Psi \rightarrow \gamma M)} \sim \frac{1}{\alpha_{s}^{2}} .
$$

The "stickiness" of a mesonic state is defined as (see, e.g., [95])

$$
S_{X}=\frac{\Gamma(J / \Psi \rightarrow \gamma X)}{\Gamma(J / \Psi \rightarrow \gamma \gamma)} .
$$

We expect the stickiness of all mesons to be comparable, while for glueballs it should be enhanced by a factor of about $S_{G} / S_{M} \approx 1 / \alpha_{s}^{4} \sim 20$,

In a recent reference [96] results of the search for $f_{J}(2220)$ production in twophoton interactions were presented. There a very small upper limit for the product of $\Gamma_{\gamma \gamma} B_{K_{s} K_{s}}$ was given, where $B_{K_{s} K_{s}}$ denotes the branching fraction of its decay into $K_{s} K_{s}$. From this it was concluded that this is a strong evidence that the $f_{J}(2220)$ is a glueball. 


\subsubsection{Heavy Quark Spectroscopy}

For charmonium production, the two-photon width $\Gamma_{\gamma \gamma}$ of $\eta_{c}\left(2960 \mathrm{MeV}, J^{P C}=\right.$ $0^{-+}$) is known from experiment. But the two-photon widths of $P$-wave charmonium states have been measured with only modest accuracy. For RHIC the study of $\eta_{c}$ is a real challenge [19]; the luminosities are falling and the branching ratios to experimental interesting channels are small.

In Table 3 (adapted from table 2.6 of [23]) the two-photon production crosssections for $c \bar{c}$ and $b \bar{b}$ mesons in the rapidity range $|Y|<7$ are given. Also given are the number of events in a $10^{6} \mathrm{sec}$ run with the ion luminosities of $4 \times 10^{30} \mathrm{~cm}^{-2} \mathrm{~s}^{-1}$ for $\mathrm{Ca}-\mathrm{Ca}$ and $10^{26} \mathrm{~cm}^{-2} \mathrm{~s}^{-1}$ for $\mathrm{Pb}-\mathrm{Pb}$. Millions of $C$-even charmonium states will be produced in coherent two-photon processes during a standard $10^{6}$ sec heavy ion run at the LHC. The detection efficiency of charmonium events has been estimated as $5 \%$ for the forward-backward FELIX geometry [23], i.e., one can expect detection of about $5 \times 10^{3}$ charmonium events in $\mathrm{Pb}-\mathrm{Pb}$ and about $10^{6}$ events in $\mathrm{Ca}-\mathrm{Ca}$ collisions. This is two to three orders of magnitude higher than what is expected during five years of LEP200 operation. Further details, also on experimental cuts, backgrounds and the possibilities for the study of $C$-even bottonium states are given in [23].

\begin{tabular}{|c|c|c|c|c|c|c|}
\hline State & Mass, & $\Gamma_{\gamma \gamma}$ & \multicolumn{2}{|c|}{$\sigma(A A \rightarrow A A+X)$} & \multicolumn{2}{c|}{ Events for $10^{6} \mathrm{sec}$} \\
\cline { 4 - 7 } & $\mathrm{MeV}$ & $\mathrm{keV}$ & $\mathrm{Pb}-\mathrm{Pb}$ & $\mathrm{Ca}-\mathrm{Ca}$ & $\mathrm{Pb}-\mathrm{Pb}$ & $\mathrm{Ca}-\mathrm{Ca}$ \\
\hline$\eta^{\prime}$ & 958 & 4.2 & $22 \mathrm{mb}$ & $125 \mu \mathrm{b}$ & $2.2 \times 10^{7}$ & $5.0 \times 10^{8}$ \\
$\eta_{c}$ & 2981 & 7.5 & $590 \mu \mathrm{b}$ & $3.8 \mu \mathrm{b}$ & $5.9 \times 10^{5}$ & $1.5 \times 10^{7}$ \\
$\chi_{0 c}$ & 3415 & 3.3 & $160 \mu \mathrm{b}$ & $1.0 \mu \mathrm{b}$ & $1.6 \times 10^{5}$ & $4.0 \times 10^{6}$ \\
$\chi_{2 c}$ & 3556 & 0.8 & $160 \mu \mathrm{b}$ & $1.0 \mu \mathrm{b}$ & $1.6 \times 10^{5}$ & $4.0 \times 10^{6}$ \\
$\eta_{b}$ & 9366 & 0.43 & $370 \mathrm{nb}$ & $3.0 \mathrm{nb}$ & 370 & 12000 \\
$\eta_{0 b}$ & 9860 & $2.5 \times 10^{-2}$ & $18 \mathrm{nb}$ & $0.14 \mathrm{nb}$ & 18 & 640 \\
$\eta_{2 b}$ & 9913 & $6.7 \times 10^{-3}$ & $23 \mathrm{nb}$ & $0.19 \mathrm{nb}$ & 23 & 76 \\
\hline
\end{tabular}

Table 3: Production cross sections and event numbers for heavy quarkonia produced in a $10^{6}$ sec run in $\mathrm{Pb}-\mathrm{Pb}$ and $\mathrm{Ca}-\mathrm{Ca}$ collisions at the $\mathrm{LHC}$ with luminosities $10^{27}$ and $4 \times 10^{30} \mathrm{~cm}^{-2} \mathrm{sec}^{-1}$. Adapted from [23].

\subsubsection{Vector-meson pair production. Total hadronic cross-section}

There are various mechanisms to produce hadrons in photon-photon collisions. Photons can interact as point particles which produce quark-antiquark pairs (jets) (see Fig. 16a), which subsequently hadronize. Often a quantum fluctuation transforms the photon into a vector meson $(\rho, \omega, \phi, \ldots)$ (VMD component) opening up all the possibilities of hadronic interactions (Fig. 16 b). In hard scattering, the structure of the photon can be resolved into quarks and gluons. Leaving a spectator jet, the quarks and gluon contained in the photon will take part in the interaction, some examples are given in Figs. 16c and d. It is of great interest to study the relative amounts of these components and their properties.

The L3 collaboration recently made a measurement of the total hadron crosssection for photon-photon collisions in the interval $5 \mathrm{GeV}<W_{\gamma \gamma}<75 \mathrm{GeV} 92$. It was found that the $\gamma \gamma \rightarrow$ hadrons cross-section is consistent with the universal Regge behavior of total hadronic cross-sections. They show a steep decrease in the region of low center of mass energy followed by a slow rise at high energies. It is parameterized as follows (see Eq. (5) of [92])

$$
\sigma_{\text {tot }}=A\left(s / s_{0}\right)^{\epsilon}+B\left(s / s_{0}\right)^{-\eta},
$$




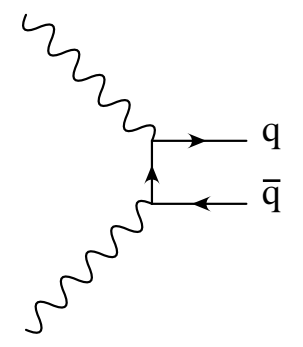

(a)
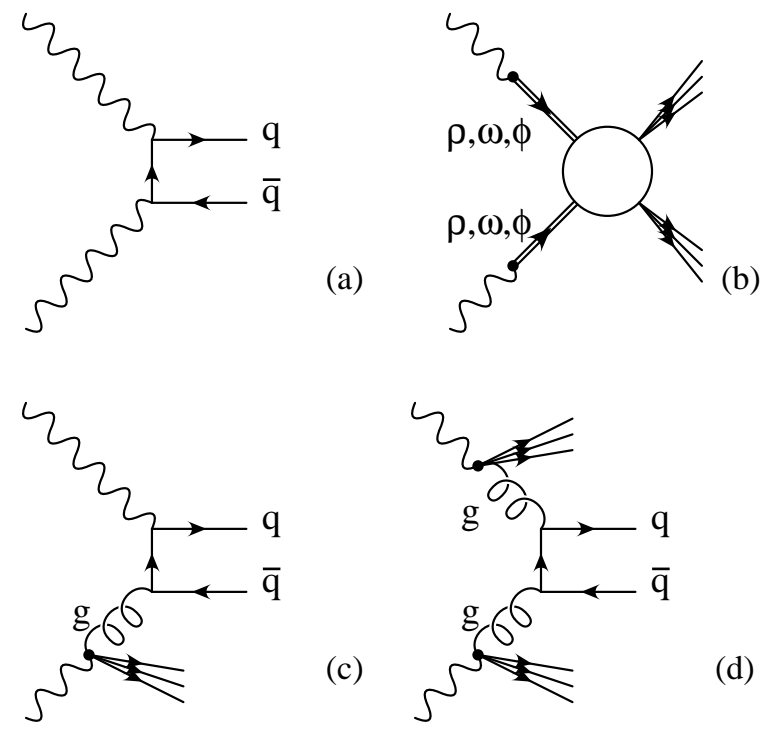

(c)

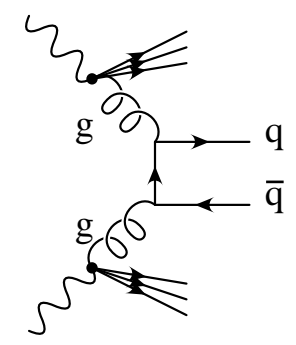

(d)

Figure 16: Diagrams showing the contribution to the $\gamma \gamma \rightarrow$ hadron reaction: direct mechanism (a), vector meson dominance (b), single (c) and double (d) resolved photons.

with $\epsilon=0.0790 \pm 0.0011, \eta=0.4678 \pm 0.0059, A=173 \pm 7 \mathrm{nb}, B=519 \pm 125 \mathrm{nb}$, and $s_{0}=1 \mathrm{GeV}^{2}$. Using this together with the effective luminosities (see Table 11), one expects about $3 \times 10^{6}$ events $/ \mathrm{GeV}$ at an invariant mass of $25 \mathrm{GeV}$ and still $4 \times 10^{4}$ events $/ \mathrm{GeV}$ at $W_{\gamma \gamma}=100 \mathrm{GeV}$.

The production of vector meson pairs can well be studied at RHIC with high statistics in the $\mathrm{GeV}$ region [18]. For the possibilities at $\mathrm{LHC}$, we refer the reader to 23 and 24, where also experimental details and simulations are described.

\section{$5.2 \gamma \gamma$-collisions as a tool for new physics}

The high flux of photons at relativistic heavy ion colliders offers possibilities for the search of new physics. This includes the discovery of the Higgs-boson in the $\gamma \gamma-$ production channel or new physics beyond the standard model, like supersymmetry or compositeness.

Let us mention here the plans to build an $e^{+} e^{-}$linear collider. Such future linear colliders will be used for $e^{+} e^{-}$, $e \gamma$ and $\gamma \gamma$-collisions (PLC, photon linear collider). The photons will be obtained by scattering of laser photons (of eV energy) on high energy electrons ( $\approx$ TeV region) (see [97). Such photons in the TeV energy range will be monochromatic and polarized. The physics program at such future machines is discussed in 98, it includes Higgs boson and gauge boson physics and the discovery of new particles.

While the $\gamma \gamma$ invariant masses which will be reached at RHIC will mainly be useful to explore QCD at lower energies, the $\gamma \gamma$ invariant mass range at LHC up to about $100 \mathrm{GeV}$ - will open up new possibilities.

A number of calculations have been made for a medium heavy standard model Higgs 99 102]. For masses $m_{H}<2 m_{W^{ \pm}}$the Higgs bosons decays dominantly into $b \bar{b}$, whereas a heavier Higgs decays into a $W^{+} W^{-}$pair. For the $\gamma \gamma \rightarrow H$ cross section we can use Eq. (71), where the two-photon width of the Higgs bosons in the standard model can be found, e.g., in [99. The calculations, using the integrated 
luminosity of Table 1, show that for Ca-Ca collisions only about one Higgs boson is produced during one year of the LHC operation. Therefore chances of finding the standard model Higgs in this case are marginal [24].

An alternative scenario with a light Higgs boson was, e.g., given in 103 in the framework of the "general two Higgs doublet model". Such a model allows for a very light particle in the few $\mathrm{GeV}$ region. With a mass of $10 \mathrm{GeV}$, the $\gamma \gamma$-width is about $0.1 \mathrm{keV}$ (see Fig. 1 of 103]). We get $2 \times 10^{3}$ events for Ca-Ca collisions, 40 for $p p$ and 8 for $\mathrm{Pb}-\mathrm{Pb}$, with the integrated luminosities of table 1 . The authors of 103 proposed to look for such a light neutral Higgs boson at the proposed low energy $\gamma \gamma$-collider. We want to point out that the LHC Ca-Ca heavy ion mode would also be very suitable for such a search.

One can also speculate about new particles with strong coupling to the $\gamma \gamma$ channel. Large $\Gamma_{\gamma \gamma}$-widths will directly lead to large $\gamma \gamma$ production cross-sections, see Eq. (71). We quote the papers [104, 105. Since the $\gamma \gamma$-width of a resonance is mainly proportional to the wave function at the origin, huge values can be obtained for very tightly bound systems. Composite scalar bosons at $W_{\gamma \gamma} \approx 50 \mathrm{GeV}$ are expected to have $\gamma \gamma$-widths of several $\mathrm{MeV}$ 104, 105. The search for such kind of resonances in the $\gamma \gamma$-production channel will be possible at LHC. Production crosssection can be directly read off from Eq. (71) and Fig. 15. E.g., take $W_{\gamma \gamma}=50 \mathrm{GeV}$ and assume a width of $\Gamma_{\gamma \gamma}=1 \mathrm{MeV}$, one obtains for a scalar particle $\left(J_{R}=0\right)$ $\sigma_{C a C a} \approx 1 M e V 10 \mathrm{pb} \mathrm{keV}^{-1}=10 \mathrm{nb}$. With an integrated luminosity of $4 \mathrm{pb}^{-1}$ in the $\mathrm{Ca}$-Ca mode, one obtains $4 \times 10^{4}$ events.

In Refs. [59, 60] $\gamma \gamma$-processes at $p p$ colliders (LHC) are studied. It is observed there that non-strongly interacting supersymmetric particles (sleptons, charginos, neutralinos, and charged Higgs bosons) are difficult to detect at the LHC. The Drell-Yan and gg-fusion mechanisms yield low production rates for such particles. Therefore the possibility of producing such particles in $\gamma \gamma$ interactions at hadron colliders is examined. Since photons can be emitted from protons which do not break up in the radiation process (see also Sec. 3) clean events can be generated which should compensate for the small number. Formula and graphs for the production of supersymmetric particles are also given in [16], where also further references can be found.

In [59] it was pointed out that at the high luminosity of $L=10^{34} \mathrm{~cm}^{-2} \mathrm{~s}^{-1}$ at the $\mathrm{LHC}(p p)$, one expects about 16 minimum bias events per bunch crossing. Even the elastic $\gamma \gamma$ events will therefore not be free of hadronic debris. Clean elastic events will be detectable at luminosities below $10^{33} \mathrm{~cm}^{-2} \mathrm{~s}^{-1}$. This danger of "overlapping events" has also to be checked for the heavy ion runs, but it will be much reduced due to the lower luminosities.

Detailed calculations have also been made for the production of a charged chargino pair via $\gamma \gamma \rightarrow \tilde{\chi}_{1}^{+} \tilde{\chi}_{1}^{-}$. The production of these charginos can be studied via their decay into a neutralino and a fermion-antifermion pair. $\tilde{\chi}_{1}^{ \pm} \rightarrow \tilde{\chi}_{1}^{0} f_{i} \bar{f}_{j}$. The most clean channel is into muons or electrons. Such an event would therefore be characterized by two fermions of opposite charge $\left(e^{+} e^{-}, \mu^{+} \mu^{-}\right.$or $\left.e^{ \pm} \mu^{\mp}\right)$ together with an unbalanced transverse momentum. In order to be able to detect the missing momentum, a closed geometry is needed. Studies were made for this process as a function of the mass of the chargino. In this case the main background - the production of a $W^{+} W^{-}$pair also decaying into two leptons of opposite charge was studied also. The cross section for this process was found to be $3.6 \mathrm{pb}$, comparable to the chargino pair production. But the harder momentum distribution of this background process can be used to distinguish it from the chargino production. Similar calculations have also been made for $p p$ collisions [60]. 


\section{Diffractive processes as background}

Diffractive processes are an important class of background to $\gamma \gamma$ final states. As the nuclei can remain intact in these collisions, they have the same signature as the photon-photon events. Therefore they cannot be distinguished from each other. Diffractive events have been studied extensively at HERA for photon-proton collisions. A future program will also study diffractive processes involving nuclei 106. Diffraction processes in $p p$ and $p \bar{p}$ are also well known from studies at the Tevatron and ISR (CERN).

Diffractive events at high energies are best described within Regge theory and in the language of the Pomeron (see, e.g., 107). It is needless to say that the possibility to study Photon-Pomeron and also Pomeron-Pomeron collisions in peripheral collisions are interesting fields in themselves. Especially photon-Pomeron fusion processes could be of interest, as they allow for final states, which are not directly possible in photon-photon events (see also the discussion about the diffractive vector-meson production in $\gamma A$-collisions in Sec. (4). Here we restrict ourselves to the estimate, how big the contribution of diffractive processes are compared to photon-photon events. Of course it is difficult to give quantitative answers at present.

A number of calculations were made within the phenomenological Dual Parton Model (DPM) [108]. These calculations 108] have been interpreted, that PomeronPomeron fusion dominates over the photon-photon cross section for almost all ions used. Only for the very heavy ions, like $\mathrm{Pb} \mathrm{Pb}$, the photon-photon process becomes comparable. Unfortunately these calculations were made without the constraint that the nuclei remain intact in the final state. As the nuclei are only weakly bound system and the nuclear interaction strong, it is very likely that a short range interaction between them leads to the breakup of the nucleus. More refined calculations have been made in the meantime [109]. The cross sections for diffractive processes are then reduced roughly by two orders of magnitude for $\mathrm{Ca}-\mathrm{Ca}$ and by three orders of magnitude for $\mathrm{Pb}-\mathrm{Pb}$ (both at LHC conditions). Only for protonproton collisions diffractive processes can be expected to dominate over photonphoton ones. Particle production from diffractive processes are also studied in [110,111. They also find that the increase of the cross section with mass number $A$ is much smaller than that for electromagnetic processes.

The problem of separating two-photon signals from background has been studied in detail for RHIC conditions in 112]. Four sources of background have been considered: Peripheral (hadronic) nucleus-nucleus collisions, beam-gas interactions, gamma-nucleus interactions and cosmic rays. In order to separate signals from background, cuts have been developed which utilize the characteristics of two-photon interactions; the most important of these cuts are multiplicity and transverse momentum. It was shown in this reference, to which we refer the reader for details, that there are high rates of $\gamma \gamma$-interactions and that the signals can well be separated from background.

\section{Electron-Positron Pair production and QED of strong fields}

Electrons (positrons) and to some extent also muons have a special status, which is due to their small mass. They are therefore produced more easily than other heavier particles and in the case of $e^{+} e^{-}$pair production lead to new phenomena, like multiple pair production. In addition the Compton wave length of the electron $(\approx 386 \mathrm{fm})$ is much larger than the size of the nuclei $(\gtrsim 7 \mathrm{fm})$. This also means that the virtuality $Q^{2}$ of the photons, which ranges from 0 up to the order of $1 / R^{2}$ 
can be much larger than the electron mass $m_{e}^{2}$. Whereas in all cases discussed above we could treat the photons as being quasireal and relate their cross section to the photon cross section, this is no longer the case here in general and therefore corrections to the EPA are needed.

The muon has a Compton wavelength of about $2 \mathrm{fm}$. This length is of the same order as the nuclear radius. We therefore expect that the EPA will give more reliable results. Both electrons and muons can be produced not only as free particles but also into an atomic states bound to one of the ions.

\subsection{Free pair production, Strong field effects and multiple pair production}

We consider the $e^{+} e^{-}$pair production in the collision of two nuclei with charges $Z_{1}$ and $Z_{2}$ and relative velocity $v$. For $v \rightarrow 0$ the electrons and positrons can adjust adiabatically to the motion of the nuclei and, with sufficiently high charge $Z_{1}$ and $Z_{2}$ one can enter a supercritical regime, where $\left(Z_{1}+Z_{2}\right) \alpha>1$. Such a situation has been studied extensively at GSI and later at Argonne; we refer the reader to the (vast) literature on this subject, see 113] where further references are given. We here study the opposite region, with $v \approx c$. A very useful and complete reference for this field is [114].

The special situation of the electron pairs can already be seen from the formula for the impact parameter dependent probability in lowest order. Using EPA one obtains [1]

$$
P^{(1)}(b) \approx \frac{14}{9 \pi^{2}}(Z \alpha)^{4} \frac{1}{m_{e}^{2} b^{2}} \ln ^{2}\left(\frac{\gamma \delta}{2 m_{e} b}\right),
$$

where $\delta \approx 0.681$ and $\gamma=2 \gamma_{c m}^{2}-1$ the Lorentz factor in the target frame, one can see that at RHIC and LHC energies and for impact parameters of the order of the Compton wave length $b \approx 1 / m_{e}$, this probability exceeds one. It was first described in [115] how unitarity can be restored by considering the production of multiple pairst.

Multiple pair production was later studied by a number of authors $117-120$ using different approximations. A general feature found by all was the fact that the probability is given to a good approximation by a Poisson distribution:

$$
P(N, b) \approx \frac{\left[P^{(1)}(b)\right]^{N}}{N !} \exp \left[-P^{(1)}(b)\right]
$$

where $P^{(1)}(b)$ is the single pair creation probability from perturbation theory, see, e.g., Eq. (76). Deviations from this Poisson form were studied in [120] and were found to be small at high energies.

The impact parameter dependence of the lowest order process was calculated in 121, 122] (see also Fig. 17), the total cross section for the one-pair production in 123, for one and multiple pair production in 124 (see Fig. 18). Of course the total cross section is dominated by the single pair production as the main contribution to the cross section comes from very large impact parameters $b$. On the other hand one can see that for impact parameters $b$ of about $2 R$ the number of electron-positron pairs produced in each ion collision is about 5 (2) for LHC with $Z=82$ (RHIC with $Z=79$ ). This means that each photon-photon event especially those at a high invariant mass - which occur predominantly at impact parameters close to $b \gtrsim 2 R$ - is accompanied by the production of several (lowenergy) $e^{+} e^{-}$pairs.

\footnotetext{
${ }^{1}$ It is interesting to remark that the fact that pair production in ion collisions grows beyond the unitarity limit was already observed by Heitler 116]. Of course at that time available energies made this only "of academic interest".
} 

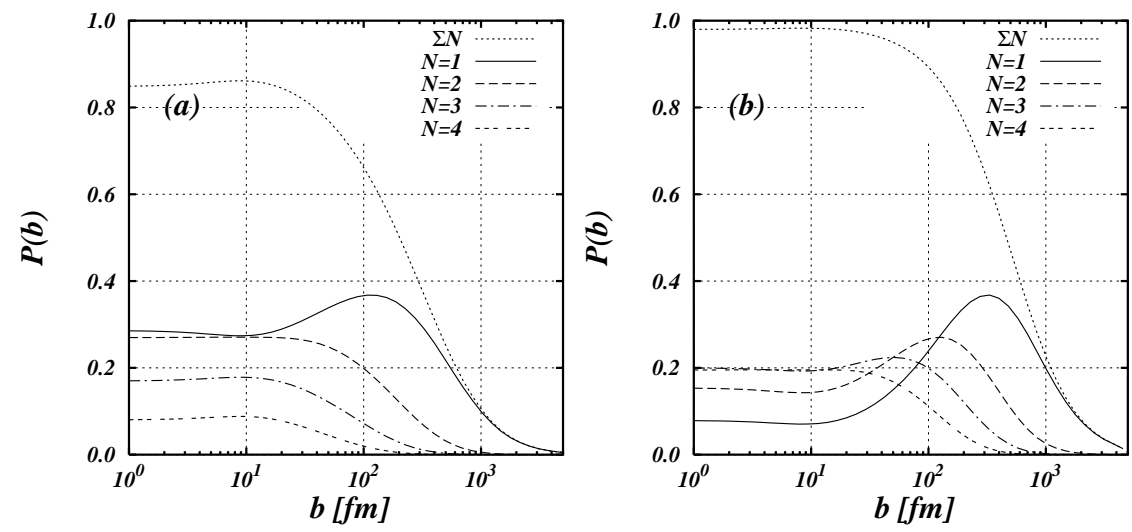

Figure 17: The impact parameter dependent probability to produce $N e^{+} e^{-}$-pairs $(N=1,2,3,4)$ in one collision is shown for both RHIC $(a, \gamma=106, A u-A u)$ and $L H C(b, \gamma=2950, P b-P b)$. Also shown is the total probability to produce at least one $e^{+} e^{-}$-pair. One sees that at small impact parameters multiple pair production can be dominant over single pair production.

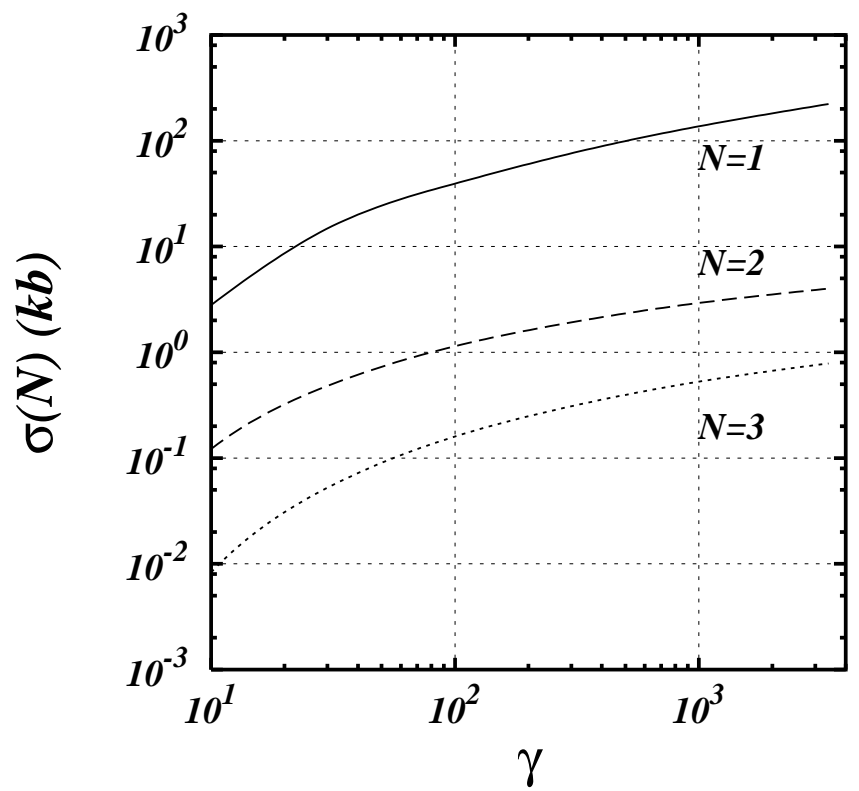

Figure 18: The total cross sections for the multiple pair production of up to three pairs is shown as a function of the Lorentz factor $\gamma$. Shown are the results for a $\mathrm{Pb}-\mathrm{Pb}$ collision. 

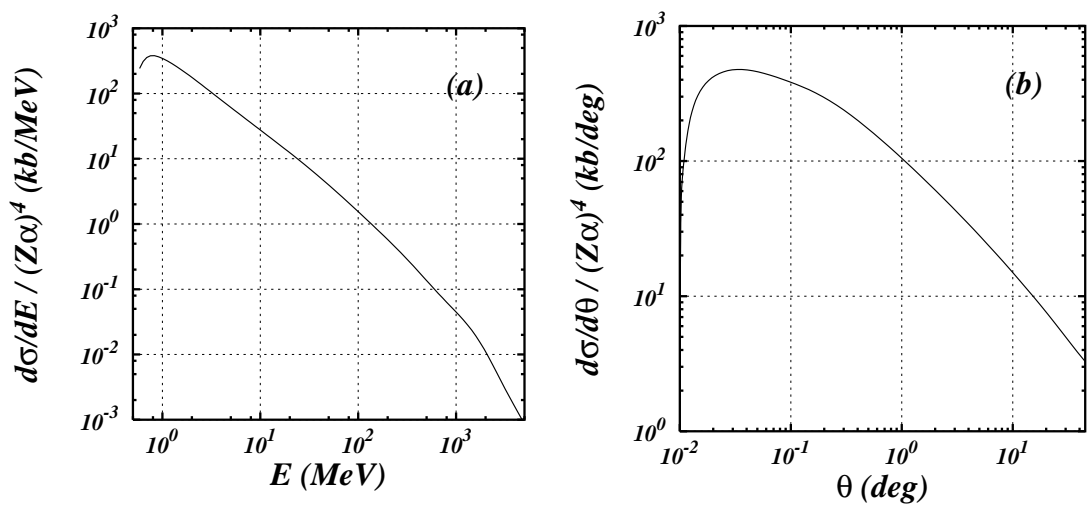

Figure 19: The single differential cross section for $e^{+} e^{-}$pair production at LHC energies. Shown are the cross sections as a function of the energy of either electron or positron (a), and as a function of the angle with the beam axis $\theta$ (b).

As the total cross section for this process is huge (about $200 \mathrm{~kb}$ for $\mathrm{Pb}$ at $\mathrm{LHC}$, $30 \mathrm{~kb}$ for $\mathrm{Au}$ at $\mathrm{RHIC}$ ), one has to take this process into account as a possible background process. Most of the particles are produced at low invariant masses (below $10 \mathrm{MeV}$ ) and into the very forward direction (see Fig. 19). Therefore most of them leave the detector along the beam pipe and are not observed. On the other hand a substantial amount of them is left also at high energies, e.g., above $1 \mathrm{GeV}$. These QED pairs also constitute a potential hazard for detectors at the colliders. In Table 1 we show the cross section for this process with the energy of either the electron or the positron above a certain threshold. Singles angular distributions of electrons (positrons) are calculated for peripheral collisions using EPA in 125. Numerical results in the relevant energy and angular ranges are presented there. The physics is discussed in terms of easy to handle analytical formulae.

\begin{tabular}{|c|rr|}
\hline$E_{t h r}(\mathrm{GeV})$ & $\sigma(\mathrm{Pb}-\mathrm{Pb}, \mathrm{LHC})$ & $\sigma(\mathrm{Ca}-\mathrm{Ca}, \mathrm{LHC})$ \\
\hline 0.25 & $3.5 \mathrm{~kb}$ & $12 \mathrm{~b}$ \\
0.50 & $1.5 \mathrm{~kb}$ & $5.5 \mathrm{~b}$ \\
1.0 & $0.5 \mathrm{~kb}$ & $1.8 \mathrm{~b}$ \\
2.5 & $0.08 \mathrm{~kb}$ & $0.3 \mathrm{~b}$ \\
5.0 & $0.03 \mathrm{~kb}$ & $0.1 \mathrm{~b}$ \\
\hline
\end{tabular}

Table 4: The cross sections of $e^{+} e^{-}$pair production with both electron and positron having an energy above a certain threshold value. Shown are results for both LHC for two different ion species.

Differential production probabilities for $\gamma \gamma$-dileptons in central relativistic heavy ion collisions are calculated using EPA and an impact parameter formulation and compared to Drell-Yan and thermal ones in 45, 126, 127. The very low $p_{\perp}$ values and the angular distribution of the pairs give a handle for their discrimination. Nuclear stopping leads to bremsstrahlung pair-production and some modification of the $\gamma \gamma$ dilepton spectra. For details we refer the reader to these references. In $[128]$ the low energy dilepton spectrum in $16 \mathrm{GeV} \pi$-p collisions was studied using the two-photon mechanism. It was found that this mechanism could not explain the experimental data 129. This is in contrast to the findings of 130. 
In the Bethe-Heitler process $\gamma+Z \rightarrow e^{+} e^{-}+Z$ higher-order effects are well known. Using Sommerfeld-Maue wave functions higher order effects are taken into account. This leads to a modification of the Born result. E.g., the total cross section (no screening) for $\omega \gg m_{e}$ is given by (see [131])

$$
\sigma=\frac{28}{9} Z^{2} \alpha r_{e}^{2}\left[\ln \frac{2 \omega}{m_{e}}-\frac{109}{42}-f(Z \alpha)\right]
$$

with the higher-order term given by

$$
f(Z \alpha)=(Z \alpha)^{2} \sum_{n=1}^{\infty} \frac{1}{n\left(n^{2}+(Z \alpha)^{2}\right)}
$$

and $r_{e}=\alpha / m_{e}$ is the classical electron radius. As far as total cross sections are concerned the higher-order contributions tend to a constant.

Using those results a modification of $e^{+} e^{-}$pair creation in $Z_{1}+Z_{2}$ collisions with respect to the lowest order result was obtained [1]. Such a treatment was not symmetrical with respect to $Z_{1}$ and $Z_{2}$ and an ad hoc symmetrization was introduced there (see Eq. (7.3.7) of [1]).

A systematic way to take leading terms of higher order effects into account in $e^{+} e^{-}$pair production is pursued in [132] using Sudakov variables and the impactfactor representation.

Nonperturbative effects are studied also in a light-front approach [133]. In this approach a gauge transformation on the time-dependent Dirac equation is performed, in order to remove the explicit dependence on the long-range part of the interaction. Similar approaches are also studied in [134, 135]. Numerical evaluation of the non-perturbative effects will be considered in a future work.

In this context the paper 136 is of interest. In this work the electromagnetic field of a particle with velocity $v$ is calculated, see e.g., the textbook result of [30]. Then the limit $v \rightarrow c$ is performed. This corresponds to the electromagnetic fields of a massless particle, which can be regarded as an "electromagnetic shock wave". The results of this paper are very much reminiscent of the sudden approximation in the semiclassical theory; for a connection of semiclassical and eikonal methods see also 137.

\subsection{Bound-Free Pair Production}

The bound-free pair production, also known as electron-pair production with capture, is a process, which is also of practical importance in the collider. It is the process, where a pair is produced but with the electron not as a free particle, but into an atomic bound state of one of the nuclei. As this changes the charge state of the nucleus, it is lost from the beam. Together with the electromagnetic dissociation of the nuclei (see Sec. 倹) these two processes are the dominant loss processes for heavy ion colliders.

In [1] an approximate value for this cross section is given as

$$
\sigma_{\text {capt }}^{K} \approx \frac{33 \pi}{10} Z_{1}^{2} Z_{2}^{6} \alpha^{6} r_{e}^{2} \frac{1}{\exp \left(2 \pi Z_{2} \alpha\right)-1}\left[\ln (\gamma \delta / 2)-\frac{5}{3}\right],
$$

where only capture to the $K$-shell is included. The cross section for all higher shells is expected to be of the order of $20 \%$ of this cross section (see Eqs 7.6.23 and 24 of [1]).

The cross section in Eq. 80 ) is of the form

$$
\sigma=A \ln \gamma+B
$$


This form has been found to be a universal one at sufficient high values of $\gamma$. The constant $A$ and $B$ then only depend on the type of the target.

The above cross section was found making use of the EPA and also using approximate wave function for bound state and continuum. More precise calculations exist 138 143] in the literature. Recent calculations within PWBA for high values of $\gamma$ have shown that the exact first order results do not differ significantly from EPA results [144, 145]. Parameterizations for $A$ and $B$ [139, 141] for typical cases are given in Table 5 .

\begin{tabular}{|c|c|c|c|c|}
\hline Ion & $A$ & $B$ & $\sigma(A u, \gamma=106)$ & $\sigma(P b, \gamma=2950)$ \\
\hline $\mathrm{Pb}$ & $15.4 \mathrm{~b}$ & $-39.0 \mathrm{~b}$ & $115 \mathrm{~b}$ & $222 \mathrm{~b}$ \\
$\mathrm{Au}$ & $12.1 \mathrm{~b}$ & $-30.7 \mathrm{~b}$ & $90 \mathrm{~b}$ & $173 \mathrm{~b}$ \\
$\mathrm{Ca}$ & $1.95 \mathrm{mb}$ & $-5.19 \mathrm{mb}$ & $14 \mathrm{mb}$ & $27.8 \mathrm{mb}$ \\
$\mathrm{O}$ & $4.50 \mu \mathrm{b}$ & $-12.0 \mu \mathrm{b}$ & $32 \mu \mathrm{b}$ & $64.3 \mu \mathrm{b}$ \\
\hline
\end{tabular}

Table 5: Parameters $A$ and B (see Eq. (81)) as well as total cross sections for the bound-free pair production for RHIC and LHC. The parameters are taken from [14].

For a long time the effect of higher order and nonperturbative processes has been under investigation. At lower energies, in the region of few $\mathrm{GeV}$ per nucleon, coupled channel calculations have indicated for a long time, that these give large contributions, especially at small impact parameters. Newer calculation tend to predict considerably smaller values, of the order of the first order result and in a recent article Baltz [146] finds in the limit $\gamma \rightarrow \infty$ that contributions from higher orders are even slightly smaller than the first order results.

The bound-free pair production was measured in two recent experiments at the SPS, at $\gamma=168$ 147 and at $\gamma \approx 2$ 148, 149. Both experiments found good agreement between measurement and calculations.

A similar process was recently used at LEAR (CERN) to produce antihydrogen. An antiproton beam with a momentum of $1.94 \mathrm{GeV} / c$ hit a Xenon target $(Z=54)$ to produce and detect antihydrogen in the bound free pair production mechanism 150. The same technique is also used at Fermilab 151, where it is also planned to measure the Lamb shift in antihydrogen as a test of CPT invariance 152, 153]. Their results are in good agreement with the recent calculations 144, 145.

We note that electron and positron can also form a bound state, positronium. This is in analogy to the $\gamma \gamma$-production of mesons ( $q \bar{q}$ states) discussed in Sec. 5 . With the known width of the parapositronium $\Gamma\left(\left(e^{+} e^{-}\right)_{n=1}{ }^{1} S_{0} \rightarrow \gamma \gamma\right)=m c^{2} \alpha^{5} / 2$, the photon-photon production of this bound state was calculated in 154. The production of orthopositronium, $n=1^{3} S_{1}$ was calculated recently [77].

As discussed in Sec. 5 the production of orthopositronium is only suppressed by the factor $(Z \alpha)^{2}$, which is not very small. Therefore one expects that both kind of positronium are produced in similar numbers. Detailed calculation show that the three-photon process is indeed not much smaller than the two-photon process 87, 81.

\section{Conclusion}

In this review the basic properties of electromagnetic processes in very peripheral hadron-hadron collisions (we deal mainly with nucleus-nucleus collisions, but proton proton collisions are also treated) are described. The method of equivalent photons is a well established tool to describe these kind of reactions. Reliable results of quasireal photon fluxes and $\gamma \gamma$-luminosities are available. Unlike electrons and 
positrons heavy ions are particles with an internal structure. We have described how to treat effects arising from this structure, and we conclude that such effects are well under control. A problem, which is difficult to judge quantitatively at the moment, is the influence of strong interactions in grazing collisions, i.e., effects arising from the nuclear stratosphere and Pomeron interactions.

The high photon fluxes open up possibilities for photon-photon as well as photonnucleus interaction studies up to energies hitherto unexplored at the forthcoming colliders RHIC and LHC. Interesting physics can be explored at the high invariant $\gamma \gamma$-masses, where detecting new particles could be within range. Also very interesting studies within the standard model, i.e., mainly QCD studies will be possible. This ranges from the study of the total $\gamma \gamma$-cross section into hadronic final states up to invariant masses of about $100 \mathrm{GeV}$ to the spectroscopy of light and heavy mesons.

We also reviewed dilepton production in very peripheral collisions. This is essentially well understood and gives rise to an experimental background. Multiple pair production is a strong field effect of principle interest. Pair production with capture is, in addition to nuclear fragmentation (sometimes called "WeizsäckerWilliams process"), a source of beam loss in the collider operation.

The future is coming soon. RHIC will be operational in only one year, LHC in approximately seven years. Therefore the planning of the experiments and necessary detectors for this kind of physics has to be done now. With the new data and new insights, that will come from these experiments, new work and theoretical understanding will be required. As an ancient motto says: "No surprise would be a surprise".

\section{Acknowledgments}

We have benefited from numerous discussions with many people over the past years. We thank all of them, especially we wish to mention A. Alscher, N. Baron, C. A. Bertulani, I. Ginzburg, O. Conradt, S. Datz, K. Eggert, R. Engel, S. Gevorkyan, M. Greiner, Z. Halabuka, S. Klein, F. Krauss, H. Meier, N. N. Nikolaev, S. Sadovsky, R. Schuch, and G. Soff.

\section{References}

[1] C. A. Bertulani and G. Baur, Phys. Rep. 163, 299 (1988).

[2] G. Baur and H. Rebel, J. Phys. G 20, 1 (1994).

[3] G. Baur and H. Rebel, Annu. Rev. Nucl. Part. Sci. 46, 321 (1996).

[4] H. Primakoff, Phys. Rev. 31, 899 (1951).

[5] R. Moshammer et al., Phys. Rev. Lett. 79, 3621 (1997).

[6] L. D. Landau and E. M. Lifshitz, Phys. Z. Sowjet. 6, 244 (1934).

[7] G. Soff, in Proc. 18th Winter School (Bielsko-Biala, Poland), 1980, edited by A. Balanda and Z. Stachura ( , , 1980), p. 201.

[8] G. Alexander, E. Gotsman, and U. Maor, Z. Phys. C 34, 329 (1987).

[9] G. Baur and C. A. Bertulani, Z. Phys. A 330, 77 (1988).

[10] M. Grabiak, B. Müller, W. Greiner, and P. Koch, J. Phys. G 15, L25 (1989). 
[11] E. Papageorgiu, Phys. Rev. D 40, 92 (1989).

[12] G. Baur, in CBPF Int. Workshop on relativistic aspects of nuclear physics, Rio de Janeiro, Brazil 1989, edited by T. Kodama et al. (World Scientific, Singapore, 1990), p. 127.

[13] G. Baur and L. G. Ferreira Filho, Nucl. Phys. A 518, 786 (1990).

[14] N. Cahn and J. D. Jackson, Phys. Rev. D 42, 3690 (1990).

[15] M. Vidović, M. Greiner, C. Best, and G. Soff, Phys. Rev. C 47, 2308 (1993).

[16] F. Krauss, M. Greiner, and G. Soff, Prog. Part. Nucl. Phys. 39, 503 (1997).

[17] F. Vannucci, in $\gamma \gamma$ Collisions, Proceedings, Amiens 1980, Vol. 134 of Lecture Notes in Physics, edited by G. Cochard (Springer, Heidelberg, Berlin, New York, 1980).

[18] S. Klein and E. Scannapieco, in Photon '97, Egmond aan Zee, edited by (World Scientific, Singapore, 1997), p. 369.

[19] S. Klein and E. Scannapieco, Coherent Photons and Pomerons in Heavy Ion Collisions, presented at 6th Conference on the Intersections of Particle and Nuclear Physics, May 1997, Big Sky, Montana, STAR Note 298, LBNL-40495, 1997.

[20] S. Klein and E. Scannapieco, STAR Note 243, 1995.

[21] S. Klein, in Photon '95, Sheffield, edited by D. J. Miller, S. L. Cartwright, and V. Khoze (World Scientific, Singapore, 1995), p. 417.

[22] K. Hencken, Yu. V. Kharlov, G. V. Khaustov, S. A. Sadovsky, and V. D. Samoylenko, TPHIC, event generator of two photon interactions in heavy ion collisions, IHEP-96-38, 1996.

[23] K. Eggert et al., FELIX Letter of Intent, CERN/LHCC 97-45, LHCC/I10, 1997.

[24] G. Baur, K. Hencken, D. Trautmann, S. Sadovsky, and Yu. Kharlov, PhotonPhoton Physics with heavy ions at CMS, CMS Note 1998/009, available from the CMS information server at http://cmsserver.cern.ch, 1998.

[25] M. M. Block and R. Cahn, Rev. Mod. Phys. 57, 563 (1985).

[26] F. Abe et al., Phys. Rev. D 50, 5550 (1994).

[27] F. Abe et al., Phys. Rev. D 50, 5535 (1994).

[28] F. Abe et al., Phys. Rev. D 50, 5518 (1994).

[29] K. R. Greider, Annu. Rev. Nucl. Part. Sci. 15, 291 (1965).

[30] J. D. Jackson, Classical Electrodynamics (John Wiley, New York, 1975).

[31] W. Nörenberg and H. A. Weidenmüller, Introduction to the theory of heavy ion collisions (Springer, Berlin, Heidelberg, New York, 1980).

[32] W. E. Frahn, Ann. Phys. 72, 524 (1972).

[33] R. M. Barnett et al., Phys. Rev. D 54, 1 (1996). 
[34] B. Z. Kopeliovich, N. N. Nikolaev, and I. K. Potashnikova, Phys. Rev. D 39, 769 (1988).

[35] R. J. Glauber, High Energy Physics and Nuclear Structure (North-Holland, Amsterdam, 1967), p. 311.

[36] J. Engel, T. K. Gaisser, P. Lipari, and T. Stanev, Phys. Rev. D 46, 5013 (1992).

[37] G. Bertsch, B. A. Brown, and H. Sagawa, Phys. Rev. C 39, 1154 (1989).

[38] G. Abbiendi, Nuovo Cim. 20, 1 (1997).

[39] R. Engel, Ph.D. thesis, Universität Gesamthochschule Siegen, 1997.

[40] V. M. Budnev, I. F. Ginzburg, G. V. Meledin, and V. G. Serbo, Phys. Rep. 15, $181(1975)$.

[41] A. Winther and K. Alder, Nucl. Phys. A 319, 518 (1979).

[42] M. Abramowitz and I. A. Stegun, Handbook of mathematical Functions (Dover, New York, 1965).

[43] G. Baur and L. G. Ferreira Filho, Phys. Lett. B 254, 30 (1991).

[44] G. Baur and N. Baron, Nucl. Phys. A 561, 628 (1993).

[45] G. Baur, Z. Phys. C 54, 419 (1992).

[46] N. Baron and G. Baur, Phys. Rev. C 49, 1127 (1994).

[47] M. Vidović, M. Greiner, and G. Soff, Phys. Rev. C 48, 2011 (1993).

[48] A. J. Baltz, M. J. Rhoades-Brown, and J. Weneser, Phys. Rev. E 54, 4233 (1996).

[49] A. J. Baltz and M. Strikman, Phys. Rev. D 57, 548 (1998).

[50] A. Baltz, C. Chasman, and S. N. White, nucl-ex/9801002, 1998.

[51] K. Hencken, D. Trautmann, and G. Baur, Phys. Rev. C 53, 2532 (1996).

[52] K. Hencken, D. Trautmann, and G. Baur, Z. Phys. C 68, 473 (1995).

[53] O. Conradt, Diplomarbeit, Universität Basel, (unpublished), 1996.

[54] B. Kniehl, Phys. Lett. B 254, 267 (1991).

[55] G. Chanfray, J. Delorme, M. Ericson, and A. Molinari, Nucl. Phys. A 556, 439 (1993).

[56] N. Baron, Ph.D. thesis, Forschungszentrum Jülich, Institut für Kernphysik Jül-2846, 1994.

[57] T. deForest and J. D. Walecka, Adv. Phys. 15, 1 (1966).

[58] F. Halzen and A. D. Martin, Quarks \& Leptons (John Wiley \& Sons, New York, 1984).

[59] M. Drees, R. M. Godbole, N. Nowakowski, and S. D. Rindami, Phys. Rev. D 50, 2335 (1994).

[60] J. Ohnemus, T. F. Walsh, and P. M. Zerwas, Phys. Lett. B 328, 369 (1994). 
[61] A. D. Martin et al., Phys. Lett. B 306, 306 (1993).

[62] A. D. Martin et al., Phys. Lett. B 309, 492 (1993).

[63] T. H. Bauer, R. D. Spital, D. R. Yennie, and F. M. Pipkin, Rev. Mod. Phys. 50, 261 (1978).

[64] J. W. Norbury and M. Waldsmith, Phys. Rev. C 57, 1525 (1998).

[65] C. Brechtmann and W. Heinrich, Z. Phys. A 330, 407 (1988).

[66] C. Brechtmann and W. Heinrich, Z. Phys. A 331, 463 (1988).

[67] P. B. Price, R. Guaxiao, and W. T. Williams, Phys. Rev. Lett. 61, 2193 (1988).

[68] I. A. Pshenichnov et al., Phys. Rev. C 57, 1920 (1998).

[69] G. Baur and C. A. Bertulani, Nucl. Phys. A 505, 835 (1989).

[70] S. Datz et al., Phys. Rev. Lett. 79, 3355 (1997).

[71] J. W. Norbury and G. Baur, Phys. Rev. C 48, 1915 (1993).

[72] J. A. Crittenden, Exclusive production of neutral vector mesons at the electron-proton collider HERA, Vol. 140 of Springer tracts in modern physics (Springer, Heidelberg, 1997).

[73] M. G. Ryskin, R. G. Roberts, A. D. Martin, and E. M. Levin, Z. Phys. C 76, 231 (1997).

[74] J. Breitweg et al., Z. Phys. C 76, 599 (1997).

[75] D. J. Miller, J. Phys. G 24, 317 (1998).

[76] D. Z. Freedman, D. N. Schramm, and D. L. Tubbs, Annu. Rev. Nucl. Part. Sci. 27, 167 (1977).

[77] I. F. Ginzburg, private communication.

[78] I. F. Ginzburg et al., Nucl. Phys. B 284, 685 (1987).

[79] I. F. Ginzburg et al., Nucl. Phys. B 296, 569 (1988).

[80] I. F. Ginzburg et al., Nucl. Phys. B 388, 376 (1992).

[81] S. R. Gevorkyan et al., hep-ph9804264.

[82] C. Hofmann, G. Soff, A. Schäfer, and W. Greiner, Phys. Lett. B 262, 210 (1991).

[83] N. Baron and G. Baur, Phys. Rev. C 48, 1999 (1993).

[84] M. Greiner et al., Phys. Rev. C 51, 911 (1995).

[85] S. Weinberg, The Quantum Theory of Fields (Cambridge University Press, Cambridge, 1997), Vol. 1.

[86] H. Kolanoski and P. Zerwas, in High Energy Electron-Positron Physics, edited by A. Ali and P. Söding (World Scientific, Singapore, 1988).

[87] Ch. Berger and W. Wagner, Phys. Rep. 176C, 1 (2987). 
[88] $\gamma \gamma$ Collisions, Proceedings, Amiens 1980, Vol. 134 of Lecture Notes in Physics, edited by G. Cochard and P. Kessler (Springer, Berlin, 1980).

[89] Proc. 9th International Workshop on Photon-Photon Collisions, San Diego (1992) (World Scientific, Singapore, 1992).

[90] Photon'95, Xth International Workshop on Gamma-Gamma Collisions and related Processes, edited by D. J. Miller, S. L. Cartwright, and V. Khoze (World Scientific, Singapore, 1995).

[91] Photon'97, XIth International Workshop on Gamma-Gamma Collisions and related Processes, Egmond aan Zee, edited by A. Buijs (World Scientific, Singapore, 1997).

[92] L3 collaboration, Phys. Lett. B 408, 450 (1997).

[93] C. N. Yang, Phys. Rev. 77, 242 (1948).

[94] S. Cooper, Annu. Rev. Nucl. Part. Sci. 28, 705 (1988).

[95] S. Cartwright et al., J. Phys. G 24, 457 (1998).

[96] R. Godang et al., Phys. Rev. Lett. 79, 3829 (1997).

[97] V. Telnov, in Photon '95, Sheffield, edited by D. J. Miller, S. L. Cartwright, and V. Khoze (World Scientific, Singapore, 1995), p. 369.

[98] I. F. Ginzburg, in Photon '95, Sheffield, edited by D. J. Miller, S. L. Cartwright, and V. Khoze (World Scientific, Singapore, 1995), p. 399.

[99] M. Drees, H. Ellis, and D. Zeppenfeld, Phys. Lett. B 223, 454 (1989).

[100] B. Müller and A. J. Schramm, Phys. Rev. D 42, 3699 (1990).

[101] E. Papageorgiu, Phys. Lett. B 352, 394 (1995).

[102] J. Norbury, Phys. Rev. D 42, 3696 (1990).

[103] D. Choudhury and M. Krawczyk, Phys. Rev. D 55, 2774 (1997).

[104] F. M. Renard, Phys. Lett. B 126, 59 (1983).

[105] U. Baur, H. Fritzsch, and H. Faissner, Phys. Lett. B 135, 313 (1984).

[106] in Future Physics at HERA, edited by G. Ingelman, A. De Roeck, and R. Klanner (DESY, Hamburg, 1996).

[107] A. H. Müller, Eur. Phys. J. A 1, 19 (1998).

[108] R. Engel et al., Z. Phys. C 74, 687 (1997).

[109] R. Engel, private communication.

[110] B. Müller and A. J. Schramm, Nucl. Phys. A 523, 677 (1991).

[111] A. J. Schramm and D. H. Reeves, Phys. Rev. D 55, 7312 (1997).

[112] J. Nystrand and S. Klein, Two Photons Physics at RHIC: Separating Signals from Backgrounds, talk presented at "Hadron'97", Brookhaven National Laboratory, August 1997, STAR Note 315, LBNL-41111 Nov.97, 1997.

[113] J. J. Griffin, The APEX/EPOS Quandary: the way out via low Energy Studies, nucl-th/9802044, 1998. 
[114] J. Eichler and W. E. Meyerhof, Relativistic Atomic Collisions (Academic Press, San Diego, 1995).

[115] G. Baur, Phys. Rev. A 42, 5736 (1990).

[116] W. Heitler, The Quantum Theory of Radiation (Oxford University Press, London, 1954).

[117] G. Baur, Phys. Rev. D 41, 3535 (1990).

[118] C. Best, W. Greiner, and G. Soff, Phys. Rev. A 46, 261 (1992).

[119] M. J. Rhoades-Brown and J. Weneser, Phys. Rev. A 44, 330 (1991).

[120] K. Hencken, D. Trautmann, and G. Baur, Phys. Rev. A 51, 998 (1995).

[121] K. Hencken, D. Trautmann, and G. Baur, Phys. Rev. A 51, 1874 (1995).

[122] M. C. Güçlü et al., Phys. Rev. A 51, 1836 (1995).

[123] C. Bottcher and M. R. Strayer, Phys. Rev. D 39, 1330 (1989).

[124] A. Alscher, K. Hencken, D. Trautmann, and G. Baur, Phys. Rev. A 55, 396 (1997).

[125] N. Baron and G. Baur, Phys. Rev. D 46, R3695 (1992).

[126] G. Baur and N. Baron, Z. Phys. C 60, 95 (1993).

[127] G. Baur, Nucl. Phys. A 538, 187c (1992).

[128] N. Baron and G. Baur, Phys. Rev. D 46, 4897 (1992).

[129] J. Stekas et al., Phys. Rev. Lett. 47, 1686 (1981).

[130] C. Bottcher, M. R. Strayer, C. J. Albert, and D. J. Ernst, Phys. Lett. B 237, 175 (1990).

[131] L. D. Landau and E. M. Lifschitz, Quantenelektrodynamik, No. IV in Lehrbuch der theoretischen Physik (Akademie Verlag, Berlin, 1986).

[132] D. Ivanov and K. Melnikov, Phys. Rev. D 57, 4025 (1998).

[133] B. Segev and J. C. Wells, Phys. Rev. A 57, 1849 (1998).

[134] U. Eichmann, J. Reinhardt, S. Schramm, and W. Greiner, nucl-th/9804064.

[135] A. J. Baltz and L. McLerran, nucl-th/9804042.

[136] R. Jackiw, D. Kabat, and M. Ortiz, Phys. Lett. B 277, 148 (1992).

[137] G. Baur, Nucl. Phys. A 531, 685 (1991).

[138] A. J. Baltz, M. J. Rhoades-Brown, and J. Weneser, Phys. Rev. A 44, 5569 (1992).

[139] A. J. Baltz, M. J. Rhoades-Brown, and J. Weneser, Phys. Rev. A 48, 2002 (1993).

[140] U. Becker, N. Grün, and W. Scheid, J. Phys. B 20, 6563 (1987).

[141] A. Aste, K. Hencken, D. Trautmann, and G. Baur, Phys. Rev. A 50, 3980 (1994). 
[142] C. K. Agger and A. H. Sørensen, Phys. Rev. A 55, 402 (1997).

[143] M. J. Rhoades-Brown, C. Bottcher, and M. R. Strayer, Phys. Rev. A 40, 2831 (1089).

[144] H. Meier et al., Eur. Phys. J. C in print, (1998).

[145] C. A. Bertulani and G. Baur, Phys. Rev. D in print, (1998).

[146] A. Baltz, Phys. Rev. Lett. 78, 1231 (1997).

[147] H. F. Krause et al., Phys. Rev. Lett. 80, 1190 (1998).

[148] A. Belkacem et al., Phys. Rev. Lett. 71, 1514 (1993).

[149] A. Belkacem et al., Phys. Rev. Lett. 73, 2432 (1994).

[150] G. Baur et al., Phys. Lett. B 368, 251 (1996).

[151] G. Blanford et al., Phys. Rev. Lett. 80, 3037 (1998).

[152] C. T. Munger, S. J. Brodsky, and I. Schmidt, Hyperfine Interactions 76, 175 (1993).

[153] C. T. Munger, S. J. Brodsky, and I. Schmidt, Phys. Rev. D 49, 3228 (1994).

[154] G. Baur, in Perspectives on Photon Interactions with Hadrons and Nuclei, Lecture Notes in Physics, edited by M. Schumacher and G. Tamas (Springer Verlag, Berlin, Heidelberg, New York, 1990), p. 111. 